\title{
Analyse des besoins non satisfaits au Sénégal
}

Kazuyo Machiyama

John C. Cleland

Follow this and additional works at: https://knowledgecommons.popcouncil.org/departments_sbsr-rh

Part of the Demography, Population, and Ecology Commons, Family, Life Course, and Society Commons, International Public Health Commons, Maternal and Child Health Commons, and the Women's Health Commons How does access to this work benefit you? Let us know!

\section{Recommended Citation}

Machiyama, Kazuyo and John C. Cleland. 2013. "Analyse des besoins non satisfaits au Sénégal," Rapport de Recherche STEP UP. London: London School of Hygiene \& Tropical Medicine. 
STEP

STRENGTHENING EVIDENCE FOR PROGRAMMING ON UNINTENDED
PREGNANCY
SENEGAL

RAPPORT

DE

RECHERCHE

MARS 2013

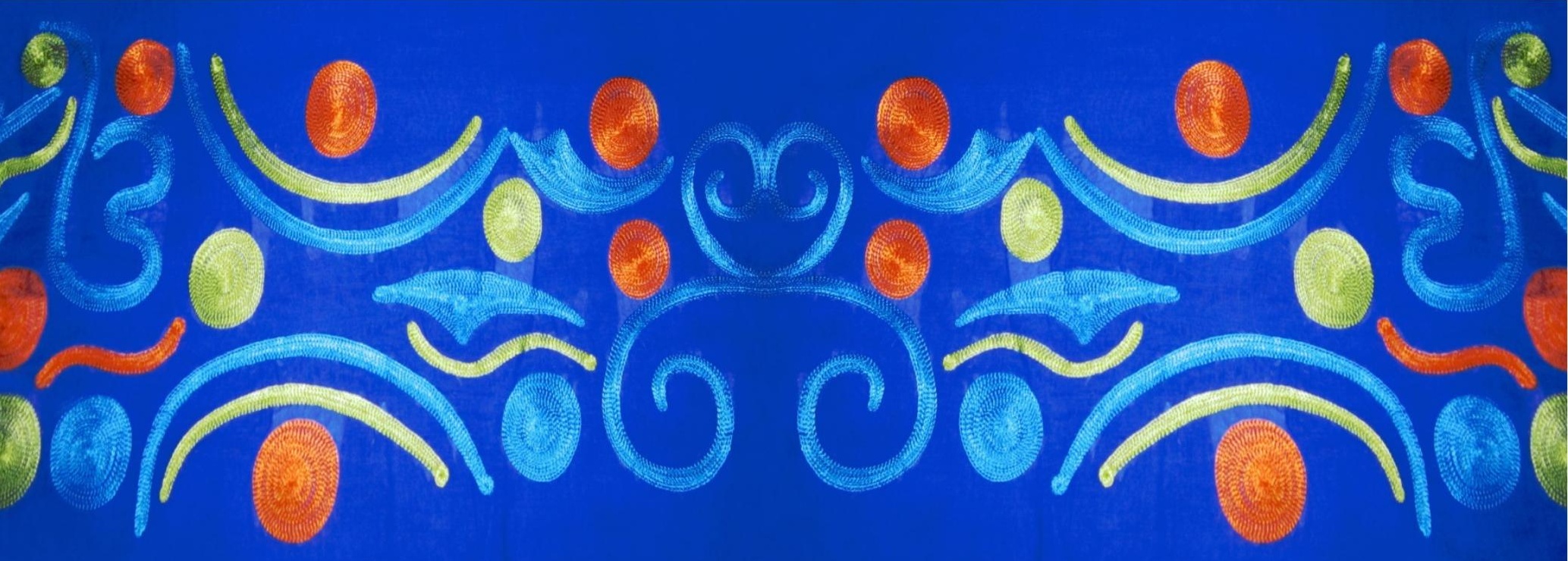

Analyse des Besoins Non Satisfaits au Sénégal

KAZUYO MACHIYAMA ET JOHN CLELAND

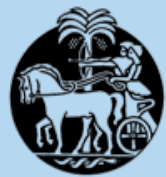




\section{Analyse des Besoins Non satisfaits au Sénégal}

KAZUYO MACHIYAMA ET JOHN CLELAND

London School of Hygiene \& Tropical Medicine

RAPPORT DE RECHERCHE STEP UP

MARS 2013 
Le Consortium du Programme de Recherche STEP UP (Consolider les Données Pour une Programmation sur les Grossesses Non-Désirées) développe des recherches pertinents aux politiques afin de promouvoir une approche basée sur les évidences pour améliorer l'accès à la planification familiale et à l'avortement sans risque. STEP UP concentre ses activités dans cinq pays : Bangladesh, Ghana, Inde, Kenya et Sénégal. STEP UP est coordonné par le Population Council en partenariat avec le African Population and Health Research Center; icddr,b; le London School of Hygiene and Tropical Medicine; Marie Stopes International; et Partners in Population and Développement. STEP UP est financé par UK aid du gouvernement britannique. www.stepup.popcouncil.org

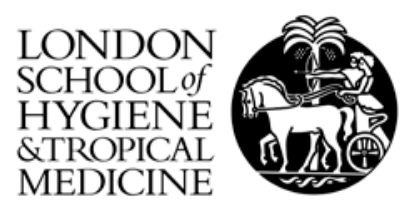

Le London School of Hygiene \& Tropical Medicine est un centre renommé mondialement pour la recherche et l'enseignement post universitaire en santé publique et mondiale. Notre mission est d'améliorer la santé et l'équité en santé au Royaume Uni et à travers le monde ; travailler en partenariat afin d'atteindre l'excellence dans la recherche en santé publique mondiale, l'éducation et la traduction des connaissances en politiques et pratique. www.Ishtm.ac.uk

\section{(2) Population Council}

Le Population Council s'attelle aux questions cruciales de santé de développement - qu'il s'agisse d'endiguer la propagation du $\mathrm{VIH}$, d'améliorer la santé reproductive et d'assurer que les jeunes mènent une vie satisfaisantes et productives. Grâce à des travaux de recherche en biomédecine, en sciences sociales et en santé publique dans 50 pays, nous travaillons avec nos partenaires pour offrir des solutions qui conduisent à des politiques, programmes et technologies plus efficaces pour améliorer la vie partout dans le monde. Fondé en 1952 et avec son siège à New York, le Council est une organisation nongouvernementale à but non lucratif avec un conseil d'administration international. www.popcouncil.org

Citation suggérée: Machiyama, K. and Cleland, J. 2013. "Analyse des besoins non satisfaits au Sénégal," Rapport de Recherche STEP UP. London: London School of Hygiene \& Tropical Medicine.

(C) 2013 London School of Hygiene \& Tropical Medicine

Veuillez contacter les co-directeurs RPC en cas de question sur STEP UP:

Dr. Harriet Birungi, hbirungi@popcouncil.org

Dr. lan Askew, iaskew@popcouncil.org

Financé par:

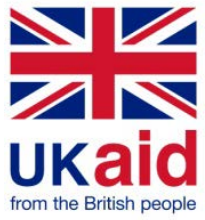




\section{Contenu}

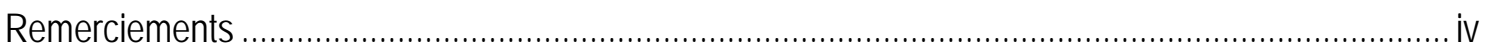

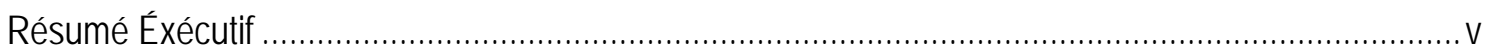

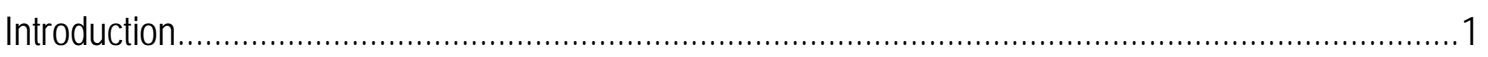

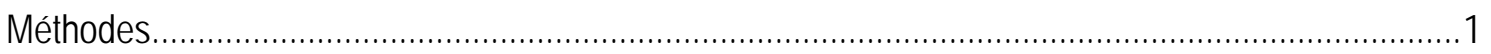

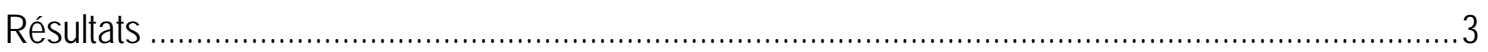

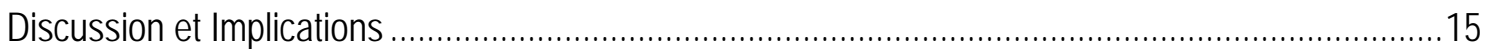

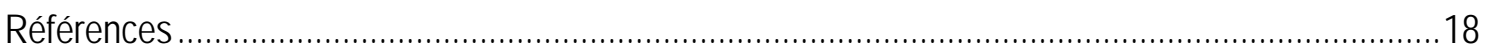

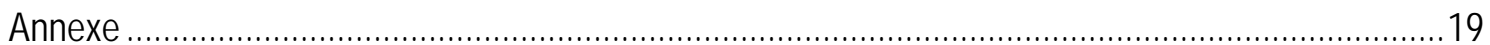




\section{Remerciements}

Nous remercions le Consortium du Programme de Recherche STEP UP (Consolider les Données Pour une Programmation sur les Grossesses Non-Désirées) pour son soutien. STEP UP est financé par UKaid du Département de Développement International. Nous voudrions remercier lan Askew, Population Council, pour ces commentaires perspicaces et pour avoir coordonné ce projet d'étude, et également, Nafissatou Diop, Population Council Sénégal, pour le partage d'idées sur les politiques et programmes. 


\section{Résumé Éxécutif}

\section{Contexte}

Depuis les 20 dernières années, les données EDS montrent que l'utilisation actuelle de la contraception moderne parmi les femmes mariées ou en cohabitation a augmenté très lentement de $5 \%$ à $12 \%$ et que les besoins non satisfaits demeurent inchangés à environ $30 \%$. La demande totale de contraception (besoins non satisfaits plus utilisation) a augmenté légèrement de $36 \%$ à $43 \%$ durant la même période. Toute avancée dans notre compréhension des causes des besoins non satisfaits pourrait avoir des implications programmatiques profondes.

\section{Objectifs}

Cette étude vise à établir l'importance relative d'un manque d'accès et d'attitudes de résistance envers l'utilisation de la contraception dans les strates géographiques et de population du Sénégal. Elle est destinée à informer les décideurs politiques sur la priorité à accorder à la communication pour le changement de comportement ou pour l'amélioration de l'accès/de l'information, et également à aider lors de la conception d'interventions pour réduire les inquiétudes par rapport à la santé et la peur des effets secondaires, tels que la provision d'une gamme de méthodes plus élargie et le counseling amélioré.

\section{Méthodes}

Les données de l'EDS Sénégal 2010-11 ont été utilisées pour l'analyse. Les analyses ont été basées sur les femmes fécondes mariées ou en cohabitation qui étaient susceptibles de tomber enceinte au moment de l'enquête. Nous avons déterminé si les femmes ayant un besoin non satisfait avaient accès (défini par la connaissance des pilules et des injectables et une source d'approvisionnement) et avec une attitude d'acceptation (définie par l'intention d'utiliser dans l'avenir). Nous avons évalué les variations des besoins non satisfaits parmi les différentes strates par des analyses bivariées et multivariées. Les raisons autodéclarées de besoin non satisfait ont été évaluées.

\section{Résultats}

Parmi les femmes susceptibles, $41 \%$ avaient un besoin non satisfait, $22 \%$ utilisaient un contraceptif modern et $36 \%$ voulaient un enfant dans les 2 ans. Celles ayant un besoin non satisfait se classaient en trois groupes principaux: ayant accès et une attitude positive; ayant accès mais pas une attitude positive ; et n'ayant pas accès. La plupart de celles n'ayant pas accès n'avaient pas l'intention d'utiliser la planification familiale. Les raisons principales auto-déclarées pour la non-utilisation étaient l'opposition de la répondante (18.6\%) et des rapports sexuels peu fréquents (17.7\%), suivi par l'allaitement et les inquiétudes par rapport à la santé. Les témoignages suggèrent que les rapports sexuels peu fréquents découlaient du fait que beaucoup de femmes n'habitaient pas dans le même ménage que leurs maris. Ceci peut être considéré également comme une alternative à la contraception. Les variations ont été considérables selon la région et le niveau d'instruction. Les moins instruites, les plus pauvres habitant dans les zones rurales, les régions du Nord, du Centre et du Sud-est étaient plus susceptibles d'avoir un besoin non satisfait sans accès ni attitude positive que leurs homologues. Les femmes allaitantes avaient un besoin non satisfait élevé. 


\section{Discussion et implications}

Plus de la moitié des femmes ayant un besoin non satisfait en planification familiale au Sénégal n'avaient pas l'intention d'utiliser la planification familiale dans l'avenir et ceci était consistant parmi toutes les strates de la population. Les raisons de non-utilisation confirment l'existence d'une opposition générale à I'utilisation de la contraception moderne. La méconnaissance et le manque d'information sont probablement à la base d'une telle opposition. Dans les sociétés avec les niveaux peu élevés d'instruction, comme au Sénégal, la suspicion initiale envers la contraception a été également documentée. Un engagement positif de la planification familiale par les leaders politiques, religieux et traditionnels pourrait aider à atténuer ces soupçons. Le fait qu'un tiers de celles ayant un besoin non satisfait n'avaient pas même l'accès de base démontrent la faible mise en œuvre des programmes dans le passé de la planification familiale au Sénégal. Les messages dans les médias, en tandem avec les initiatives communautaires d'information peuvent réduire le manque de connaissance sur les méthodes et les sources d'approvisionnement. Le niveau élevé de besoin non satisfait parmi les femmes allaitantes nécessitent un plus grand focus sur la contraception postpartum. 


\section{Introduction}

La majorité des grossesses non désirées découle de besoin non satisfait de contraception et l'objectif primordial des programmes de planification familiale est de réduire ces besoins non satisfaits. Selon l'EDS du Sénégal en 2010-11, un tiers des femmes en âge de procréer ont déclaré un besoin non satisfait. Les avancées dans notre compréhension des causes de ces besoins non satisfaits pourraient avoir des implications profondes sur le plan d'action du gouvernement sénégalais de faire accroître la prévalence contraceptive de 12\% en 2012 à 27\% en 2015.

L'objectif principal de ce projet est d'établir l'importance relative du manque d'accès et de l'attitude de résistance envers l'utilisation de la planification familiale dans la comptabilisation des besoins non satisfaits dans les différentes strates de population du Sénégal. Les résultats peuvent être utiles pour les décideurs politiques pour décider de la priorité qui devrait être accordée à la communication pour le changement de comportement ou l'amélioration de l'accès/de l'information pour les différentes strates socio-économiques et pour les différentes régions géographiques. Ils pourraient également être utiles pour les interventions visant à réduire les inquiétudes par rapport à la santé et la peur des effets secondaires tels que la provision d'une gamme de méthodes plus élargie et le counseling amélioré. Ce rapport présente les résultats des analyses faites à partir des dernières données EDS.

\section{Méthodes}

\section{Données}

Les données de l'EDS Sénégal 2010-11 ont été utilisées pour l'analyse. Les femmes qui soit ne veulent plus d'enfant soit n'en veulent pas dans les deux ans mais n'utilisent pas de méthodes de contraception sont considérées comme ayant un besoin non satisfait en contraception. Une définition standard du besoin non satisfait a été utilisée selon le rapport 2012 de Bradley et al sur les besoins non satisfaits (Bradley et al. 2012). Cependant, les femmes enceintes ou qui étaient toujours en abstinence ou en aménorrhée postpartum après l'accouchement de leur plus récent enfant, ont été exclues car elles n'étaient pas exposées au risque de conception au moment de l'enquête. Les femmes déclarant avoir été stérilisées ont été classées comme utilisatrice d'une méthode moderne de limitation. Les femmes qui n'étaient pas en union ont été également exclues car leur profil d'utilisation de méthode est très différent de celui des femmes mariées ou en union et donc l'accès ne peut pas être mesuré de la même manière pour les deux groupes. En fait, les préservatifs sont leur méthode principale tandis que la méthode est rarement utilisée par les femmes mariées. L'usage des préservatifs représentait $44 \%$ de l'utilisation de méthode moderne parmi les célibataires, mais seulement $5 \%$ parmi les femmes actuellement mariées en 2010-11 (Agence Nationale de la Statistique et de la Démographie (ANSD) [Sénégal] and ICF International 2012).

\section{Besoins non satisfaits en planification familiale}

L'analyse principale est de décomposer le besoin non satisfait en planification familiale et d'établir l'importance relative du manque d'accès et de l'attitude de résistance envers la planification familiale. Ansley Coale (1973) a suggéré qu'une transition durable et rapide de la fécondité nécessite qu'une grande portion de la population soit 'prête, volontaire et capable' d'utiliser les contraceptifs. Suite aux modifications de Lesthaeghe et Vanderhoeft (2001), Cleland et al. (2011) ont développé de mesures innovantes pour évaluer le degré auquel les populations disposeront de ces trois pré-conditions pour l'utilisation et pour mesurer les tendances. Nous avons étendu et adapté l'approche utilisée dans l'étude 
sur le progrès en besoin, accès et attitude envers la planification familiale en Afrique. (Cleland et al. 2011). Cette étude a développé une nouvelle mesure d'accès physique: la connaissance des deux méthodes modernes les plus populaires en Afrique Subsaharienne, i.e. les pilules et l'injectable, et la connaissance d'une source d'approvisionnement en planification familiale. Cette mesure n'est pas idéale pour deux raisons principales. D'abord, connaître les méthodes peut inclure de l'information erronée. Deuxièmement, la restriction aux pilules et à l'injectable est évidemment une mesure partielle de connaissance des méthodes contraceptives mais est justifiée par le fait qu'elle représente trois quarts de toute utilisation de méthode moderne au Sénégal, et, parmi les non-utilisatrices qui ont l'intention d'utiliser dans l'avenir, seulement 15\% mentionnent des alternatives de longue durée telles que la stérilisation, le DIU ou les implants comme étant leur méthode de préférence selon l'EDS Sénégal 2010-11. Connaître une source n'est pas une mesure idéale non plus car cela ne nous dit rien sur le temps de trajet ou la distance. Néanmoins, connaître les deux méthodes principales et où les obtenir résume les deux composantes les plus fondamentaux de l'accès.

L'étude de Cleland et al. de 2011 a également utilisé les réponses aux questions sur l'approbation de la planification familiale en tant que mesure d'attitude mais ces questions ont été omis de la plus récente série d'EDS. Une analyse préliminaire a montré que l'approbation d'une femme a été associée à son intention d'utiliser dans l'avenir. Lors de l'EDS 2005 du Sénégal, 51.3\% des sénégalaises qui ont approuvée la planification familiale avaient l'intention d'utiliser dans l'avenir comparé à seulement $3.6 \%$ de celles qui désapprouvaient. Manifestement, l'approbation et l'intention ne sont pas identiques, peut-être en partie parce que certaines qui approuvent ne prévoient aucun besoin dans l'avenir et de ce fait, n'ont pas l'intention d'utiliser. Néanmoins, $91 \%$ de celles qui ont l'intention d'utiliser approuvaient la planification familiale. II semble donc logique d'interpréter l'intention comme indicateur d'attitude pour les fins de ce rapport.

Basé sur ces deux mesures, nous avons identifié si les femmes ayant un besoin non satisfait de planification familiale avaient également accès à, et une attitude d'acceptation envers, la contraception. Nous avons divisé la population de l'étude en 7 groupes: (a) besoin non satisfait: accès et attitude positive; (b) besoin non satisfait: accès, mais pas d'attitude positive; (c) besoin non satisfait: attitude positive, mais pas accès; (d) besoin non satisfait: ni accès ni attitude positive, (e) utilisant n'importe quelle méthode pour l'espacement; ( $f$ ) utilisant n'importe quelle méthode pour limiter; and ( $\mathrm{g}$ ) désireuse d'avoir un autre enfant dans les 2 ans et n'utilisant pas de méthode moderne. Les catégories (e) et (f) représentent un besoin satisfait tandis que la catégorie $(\mathrm{g})$ indique aucun besoin. Les définitions détaillées se trouvent dans le Tableau A en Annexe.

La question sur la connaissance d'une source de planification familiale a été demandée uniquement aux femmes qui n'utilisaient aucune méthode de planification familiale, y compris les méthodes traditionnelles. Puisqu'il n'a pas été demandé aux utilisatrices de méthodes traditionnelles si elles connaissaient une source de planification familiale, elles ont été exclues de l'analyse. Dans les données EDS Sénégal 2010-11, ces femmes comptaient pour moins de 1\% des femmes fécondes mariées ou en cohabitation qui étaient susceptibles de devenir enceinte. 
Les informations sur l'utilisation antérieure d'une méthode moderne est utile pour distinguer entre les femmes qui ont une expérience avec la contraception mais qui ont peut-être abandonné de celles qui n'ont aucune expérience. L'EDS 2010-11 au Sénégal a recueilli un calendrier contraceptif, ce qui permet la dérivation d'une mesure approximative de l'utilisation antérieure d'une méthode moderne ${ }^{1}$. Si une femme a utilisé une méthode contraceptive dans n'importe quel mois durant la période couverte par le calendrier (entre janvier 2005 et le mois de l'entretien), la méthode a été prise en compte. Parmi les femmes ayant un besoin non satisfait, seulement $16 \%$ avaient utilisé une méthode moderne dans les 5 années précédentes.

\section{Strates de la Population}

Nous avons évalué les variations des besoins non satisfaits pour différentes strates géographiques et socio-économique (résidence, zone géographique, âge et niveau d'instruction de la répondante, richesse du ménage, mesure combinée de richesse et de résidence urbain-rural, mesure combinée d'éducation et résidence) et le statut reproductif (parité, récence du dernier accouchement, le statut d'allaitement, utilisation antérieure d'une méthode moderne).

\section{Analyse}

Nous avons d'abord évalué les associations entre le besoin non satisfait en planification familiale et les différentes strates de population/géographiques du Sénégal par une analyse bivariée en s'appuyant sur le test $\mathrm{du} x^{2}$. Ensuite, les analyses de régression logistique multivariée et de régression logistique multinomiale ont été utilisées pour estimer les probabilités ajustées d'avoir un besoin non satisfait dans différentes strates de la population. En outre, les raisons de non-utilisation de la planification ont été explorées.

\section{Résultats}

L'analyse est basée sur 4663 femmes mariées ou en cohabitation susceptibles à devenir enceinte qui n'utilisaient pas de méthode traditionnelle (voir Tableau A.2). Ces femmes comprenaient $45 \%$ de toutes les femmes actuellement mariées ou en cohabitation. Des proportions importantes de femmes mariées ou en cohabitation ont été exclues à cause d'une grossesse actuelle (7.4\%), d'une aménorrhée postpartum (14.3\%) et d'infertilité (11.5\%).

\footnotetext{
${ }^{1}$ Les questions à savoir si une femme a déjà utilisé des méthodes de planification familiale spécifiques ont été exclues de la Phase 6 de l'EDS. Au lieu de cela, il a été demandé à la femme 'Avez-vous déjà utilisé quelque chose ou essayé de retarder ou d'éviter de devenir enceinte?'. Mais les données sur l'utilisation mensuelle de la planification familiale au cours des 5 dernières années sont disponibles dans le calendrier.
} 
Figure 1: Besoin non satisfait, utilisation d'une méthode moderne et désir de fécondité Sénégal, 2010-11

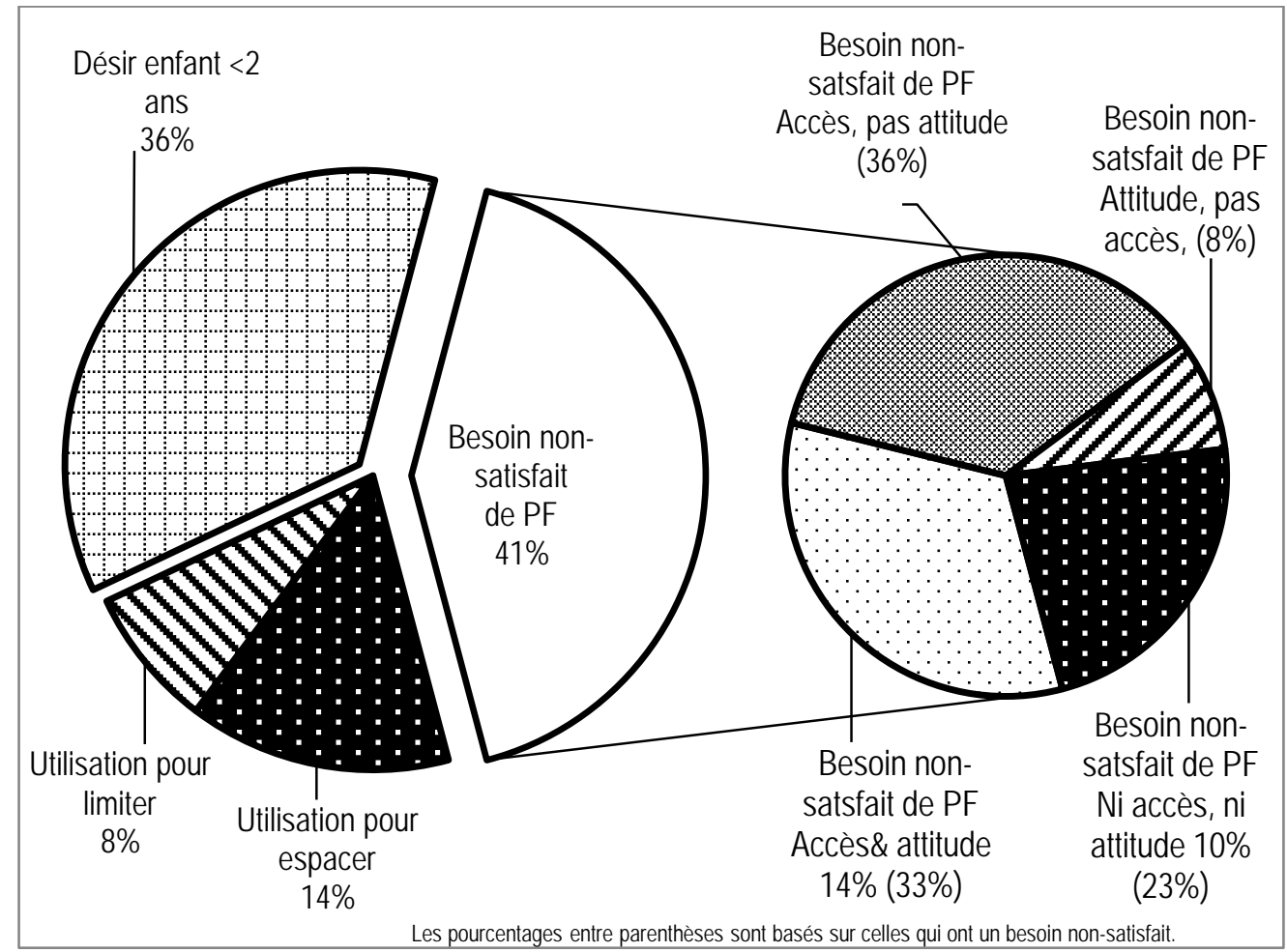

La Figure 1 présente les proportions de femmes ayant un besoin non satisfait, utilisant une méthode moderne ou désirant un enfant au cours des 2 prochaines années. Plus de $40 \%$ des femmes avaient un besoin non satisfait en planification familiale, uniquement $22 \%$ utilisaient les contraceptives modernes soit pour espacer soit pour limiter et 36\% souhaitaient un enfant au cours des 2 prochaines années². Parmi celles ayant un besoin non satisfait en planification familiale, $36 \%$ ont été classées comme ayant l'accès mais pas d'attitude positive, un tiers comme ayant l'accès et une attitude positive et un autre tiers manquait l'accès. Ainsi, une majorité $(69 \%)$ avaient l'accès, et environ la moitié des ces femmes avaient une attitude positive. Parmi les $31 \%$ sans accès, la majorité avaient une attitude défavorable. La résistance à l'utilisation de la contraception semble être généralisée.

Les inégalités de besoins non satisfaits parmi les différentes strates de population et géographiques ont été significatives. Le Tableau A.3 représente les distributions des besoins non satisfaits, de la préférence en matière de fécondité et de l'utilisation d'une méthode moderne par strate. En particulier, le besoin non satisfait a été nettement plus élevé parmi les résidentes rurales, les femmes plus âgées, les femmes non instruites et les plus pauvres.

\footnotetext{
2 Les méthodes traditionnelles et folkloriques (y compris la méthode d'aménorrhée lactationelle (MAMA)) ne sont pas considérées comme des méthodes modernes. La MAMA est parfois considéré comme une méthode moderne, mais dans cette enquête uniquement une femme exposée a signalé MAMA.
} 
II existe également des variations régionales significatives (voir Carte en Figure A.1 de l'Annexe). Cependant, l'impression dominante est que le besoin non satisfait est élevé dans toutes les régions, y compris la région occidentale où se trouve la capitale, Dakar. Les besoins non satisfaits varient de $35 \%$ dans le Sud à $47 \%$ dans la région Nord (voir Tableau A.3 et Figure 2). L'accès et l'attitude d'acceptation varient entre régions. Quarante-deux pourcent des femmes ayant un besoin non satisfait dans le sud avaient l'accès et une attitude favorable. Par contre, uniquement $26 \%$ des femmes dans le sud-est avaient l'accès et l'attitude et la majorité (35\%) n'avaient ni l'accès ni l'attitude positive.

Figure 2: Besoin non satisfait en planification familiale par zone géographique

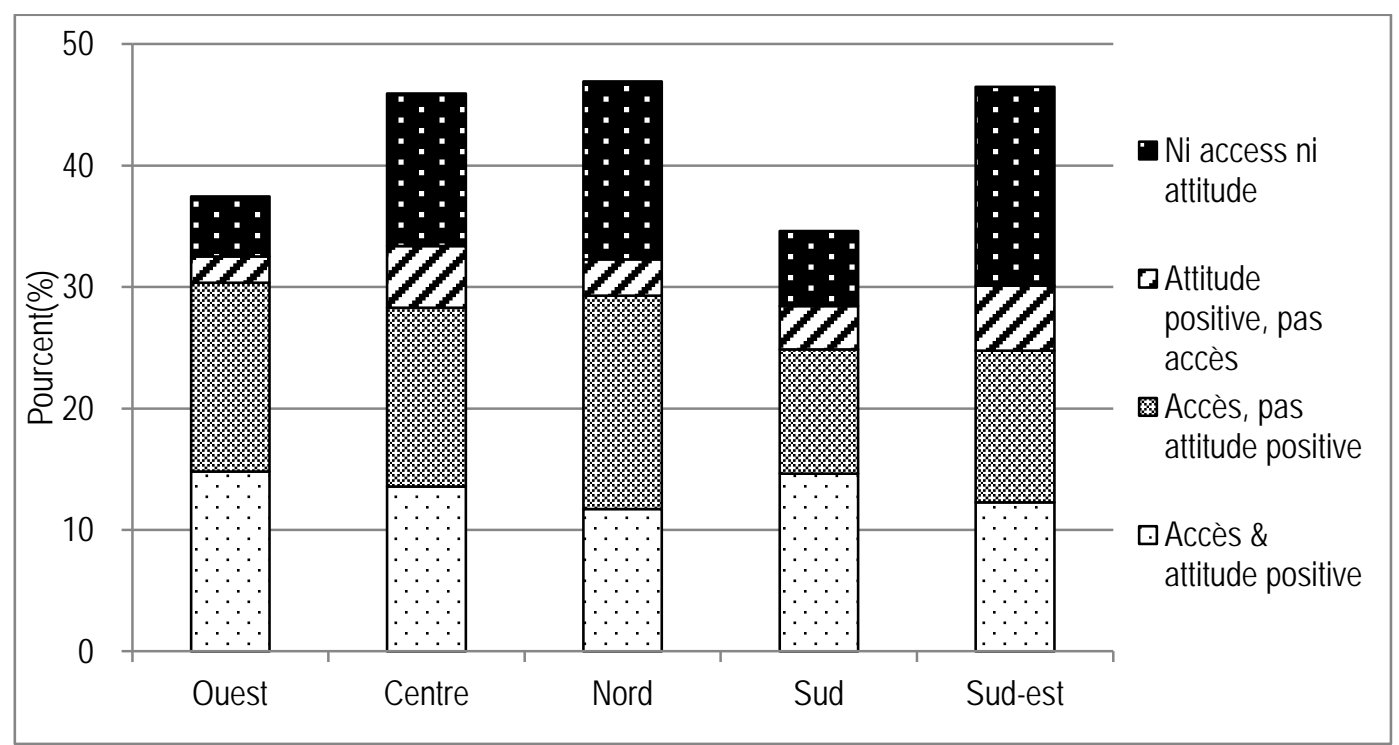

Figure 3: Besoin non satisfait en planification familiale par instruction

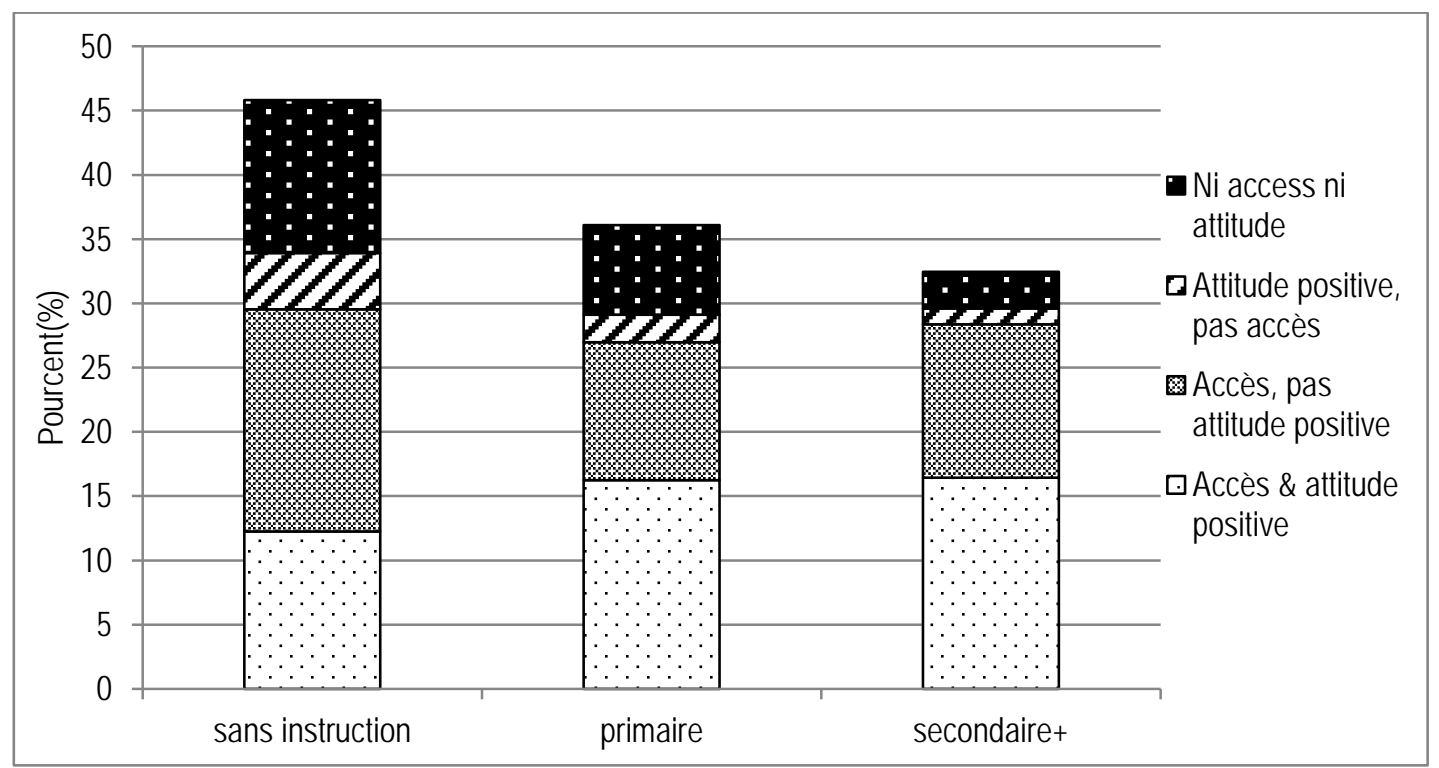


Les inégalités au niveau de l'instruction étaient évidentes. La majorité (63\%) des femmes mariées ou en cohabitation susceptibles de tomber enceinte n'avaient aucune instruction, $24 \%$ avaient fait l'école primaire et $13 \%$ l'école secondaire ou plus. Parmi celles sans instruction, $46 \%$, comparé à environ $33 \%$ de celles avec une instruction niveau primaire ou plus, avaient un besoin non satisfait (Figure 3). L'attitude de résistance était particulièrement élevée parmi les femmes sans instruction : $64 \%$ des femmes ayant un besoin non satisfait et aucune instruction contre $50 \%$ de celles avec une instruction de niveau primaire n'avaient aucune intention d'utiliser une méthode contraceptive dans l'avenir. Le manque d'accès était également notablement plus élevé chez celles sans instruction que chez les autres.

Les grandes différences urbain-rural étaient également évidentes (Tableau A.3). Presqu'un tiers (32\%) des femmes urbaines utilisaient une méthode moderne contre seulement $12 \%$ des femmes rurales. La différence en besoin non satisfait était plus étroite (38\% contre $45 \%$ ) parce qu'il était plus probable que les femmes rurales souhaitent avoir un enfant plus tôt que leurs homologues urbaines (29.5\% contre $42.4 \%)$.

A la page 24 de ce rapport, les distributions du besoin non satisfait sont représentées par une variable combinant éducation et résidence. Comme attendu, il existe un gradient éducatif dans le besoin non satisfait aussi bien dans les zones urbaines que rurales, mais, même parmi la petite élite de femmes urbaines ayant été à école secondaire, $32 \%$ avaient un besoin non satisfait. Peu de personne de cette élite manquait d'accès, mais $43 \%$ déclaraient n'avoir aucune intention d'utiliser une méthode contraceptive dans l'avenir.

Figure 4: Besoin non satisfait en planification familiale par richesse du ménage

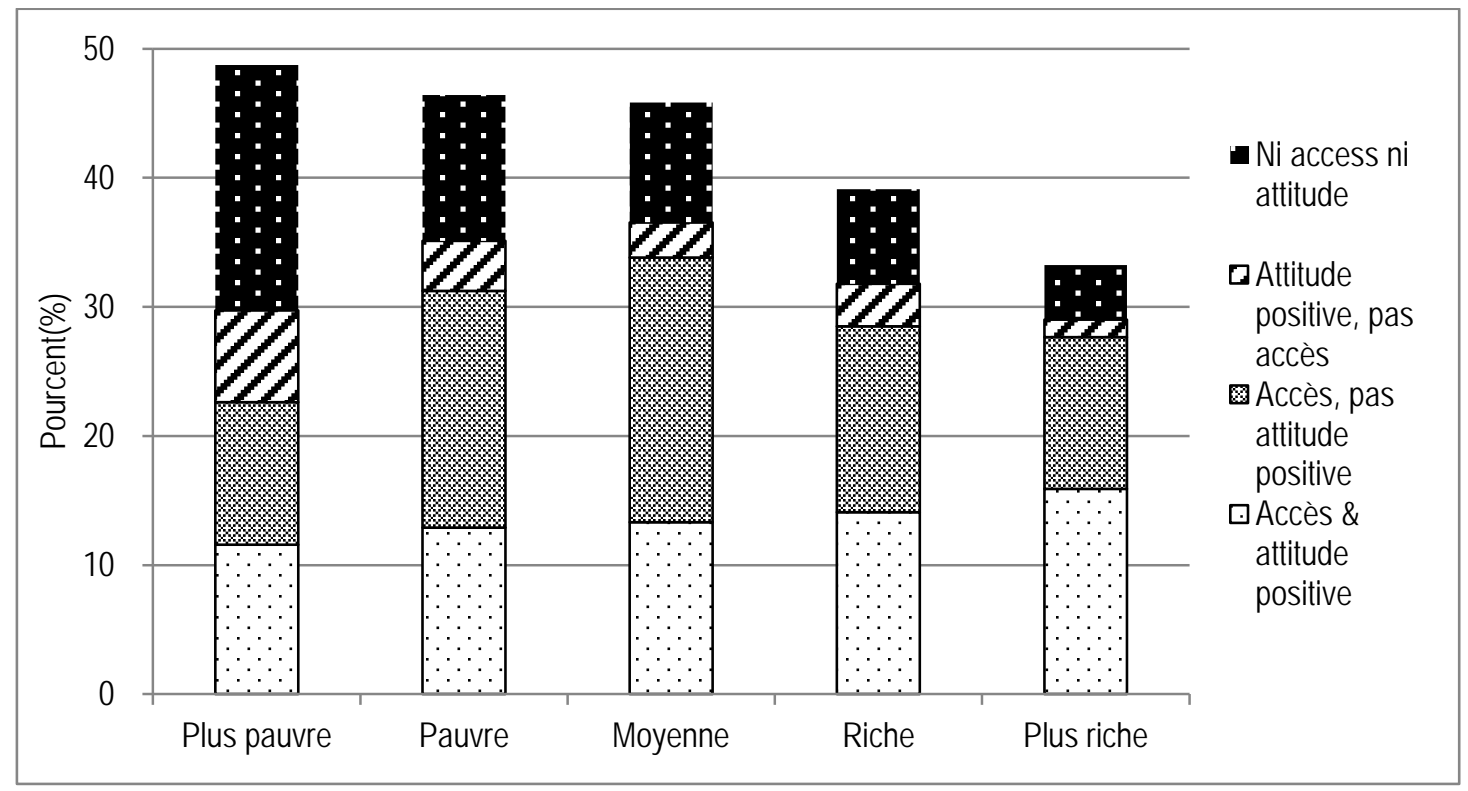


La Figure 4 démontre des gradients nets de besoin non satisfait parmi les quintiles de richesse. Environ la moitié des femmes dans la quintile la plus pauvre comparé à seulement un tiers dans la quintile la plus riche avaient un besoin non satisfait. Les femmes qui n'ont ni l'accès ni l'attitude étaient concentrées de manière disproportionnée dans la quintile la plus pauvre. Trente-neuf pourcent d'entre elles n'avaient ni l'accès ni l'attitude favorable pour l'utilisation de la planification familiale.

Figure 5: Besoin non satisfait en planification familiale depuis le dernier accouchement

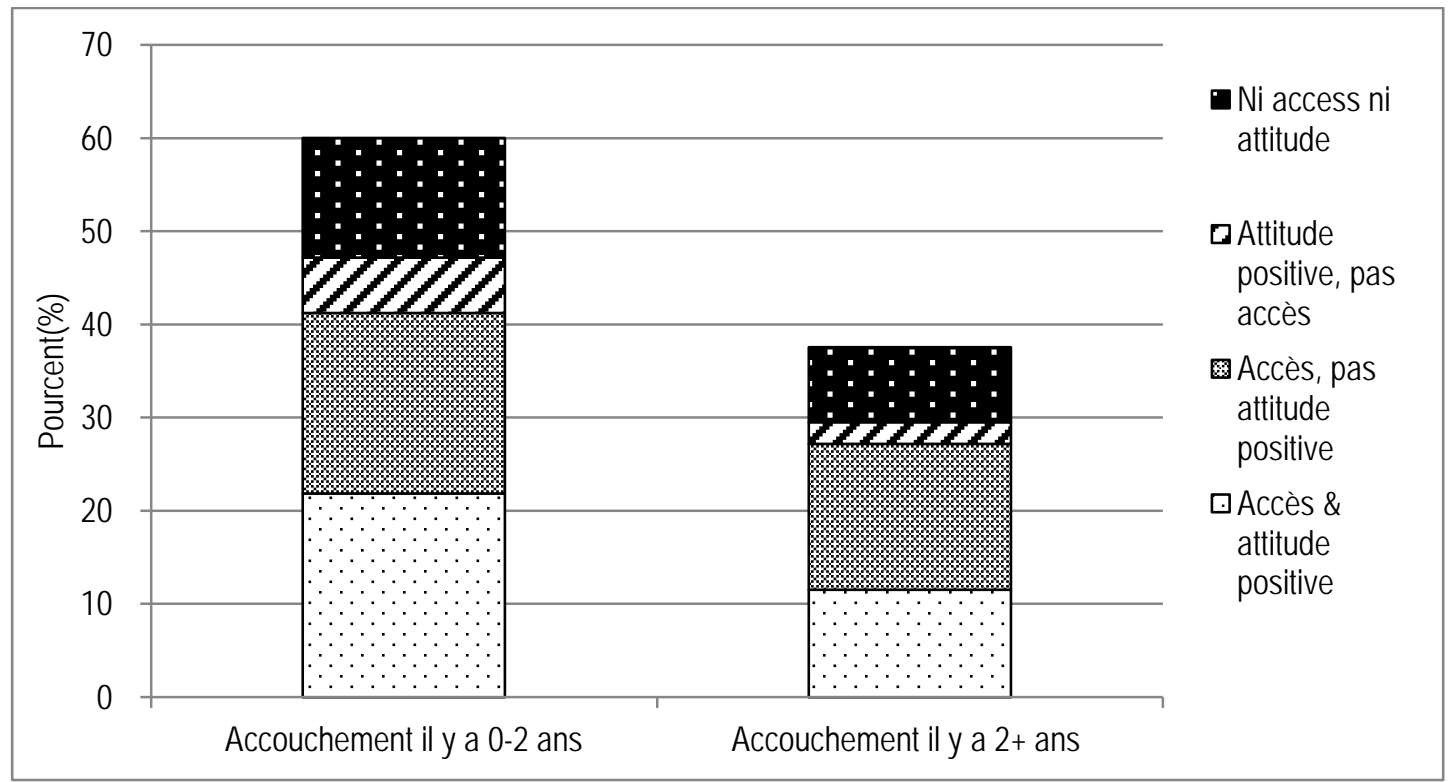

Un des groupes ayant un besoin non satisfait des plus élevés était les femmes qui avaient accouché dans les 2 ans avant l'enquête. $60 \%$ d'entre elles ont signalé un besoin non satisfait comparé à $37.6 \%$ parmi les femmes qui avaient accouché depuis plus de 2 ans (voir Figure 5). Plus de $36 \%$ des femmes en postpartum ayant un besoin non satisfait n'avaient apparemment aucun problème d'accès ou d'attitude. 
Figure 6: Besoin non satisfait en planification familiale selon l'état d'allaitement parmi les femmes ayant accouché au cours des 2 derniers années

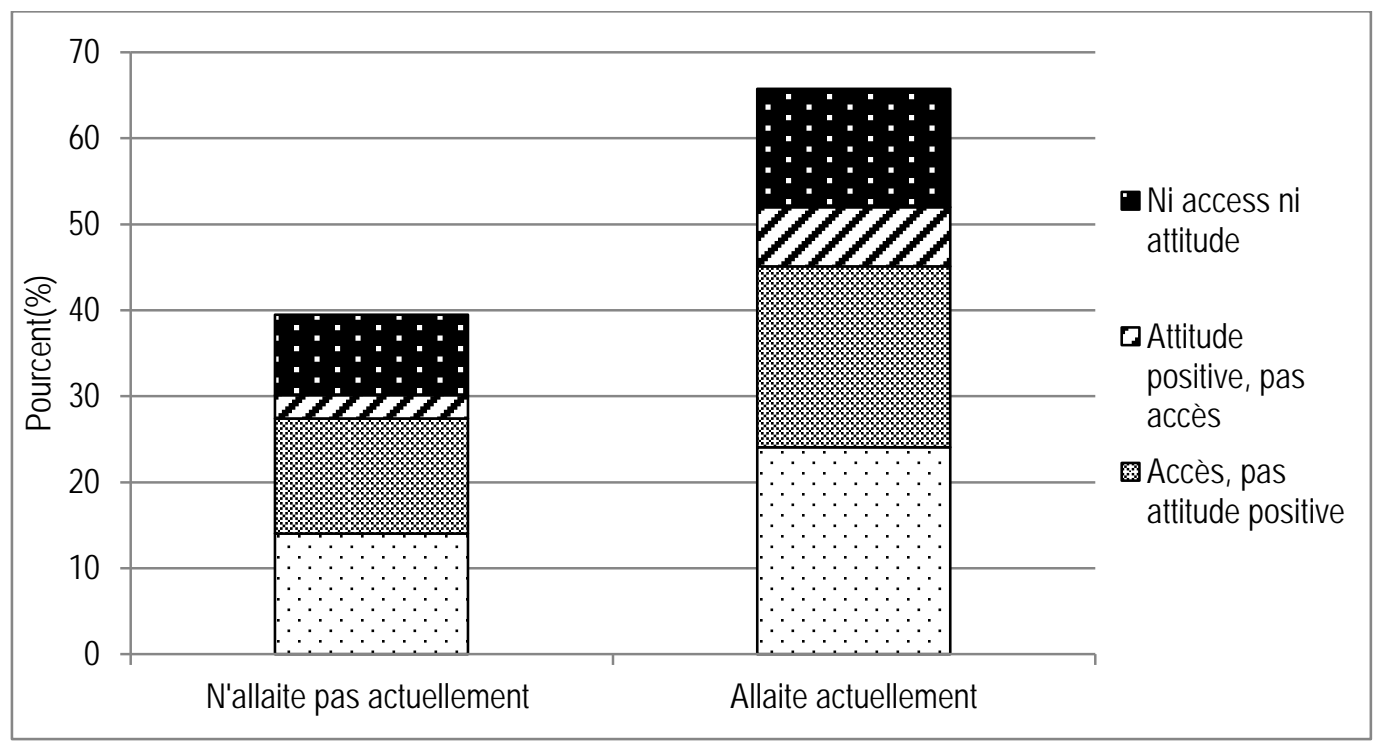

Comme présenté dans la Figure 6, parmi les femmes ayant accouché au cours des 2 ans précédents l'enquête, on constate une différence importante dans le niveau de besoin non satisfait entre les femmes allaitantes et les femmes qui avaient sevré leur enfant: $66 \%$ des femmes allaitantes contre $40 \%$ des femmes qui n'allaitaient pas avaient un besoin non satisfait. La raison de cette différence est que ces dernières étaient plus susceptibles de vouloir un autre enfant dans les 2 années à venir (36.4\%) que celles qui allaitaient encore (9.5\%). Les différences entre les deux groupes dans l'utilisation actuelle étaient négligeables (voir Tableau A.3). Parmi les femmes qui avaient accouché depuis plus de 2 ans, la minorité qui allaitaient encore, avaient un besoin non satisfait plus élevé que les autres.

Le besoin non satisfait différait également selon la parité. Comme présenté dans la Figure 7, 56\% des femmes avec 5 enfants ou plus avaient un besoin non satisfait comparé à 33\% parmi les femmes ayant 0 - 2 enfants. La plupart des femmes avec une parité élevée avaient l'accès, mais l'attitude favorable manquait. De petites différences en besoin non satisfait selon le groupe d'âge ( $<30$ ans vs $30+$ ans) existent également.

Le besoin non satisfait était extrêmement élevé (63\%) parmi le nombre relativement petit de femmes qui ne voulaient plus d'enfant (Tableau A.3). Cependant, puisque le désir d'espacer ou de retarder la maternité est beaucoup plus commun que le désir d'arrêter complètement, deux tiers des besoins non satisfaits au Sénégal découlent de la non-utilisation parmi les femmes souhaitant retarder la prochaine naissance.

La majorité des femmes ayant un besoin non satisfait n'ont pas eu d'expérience avec l'utilisation de méthodes modernes au cours des cinq dernières années et la plupart n'en ont probablement jamais utilisé. Celles qui en ont déjà utilisé ne représentaient que $16 \%$ des besoins non satisfaits (Tableau A.3). 
Figure 7: Besoin non satisfait en planification familiale selon la parité

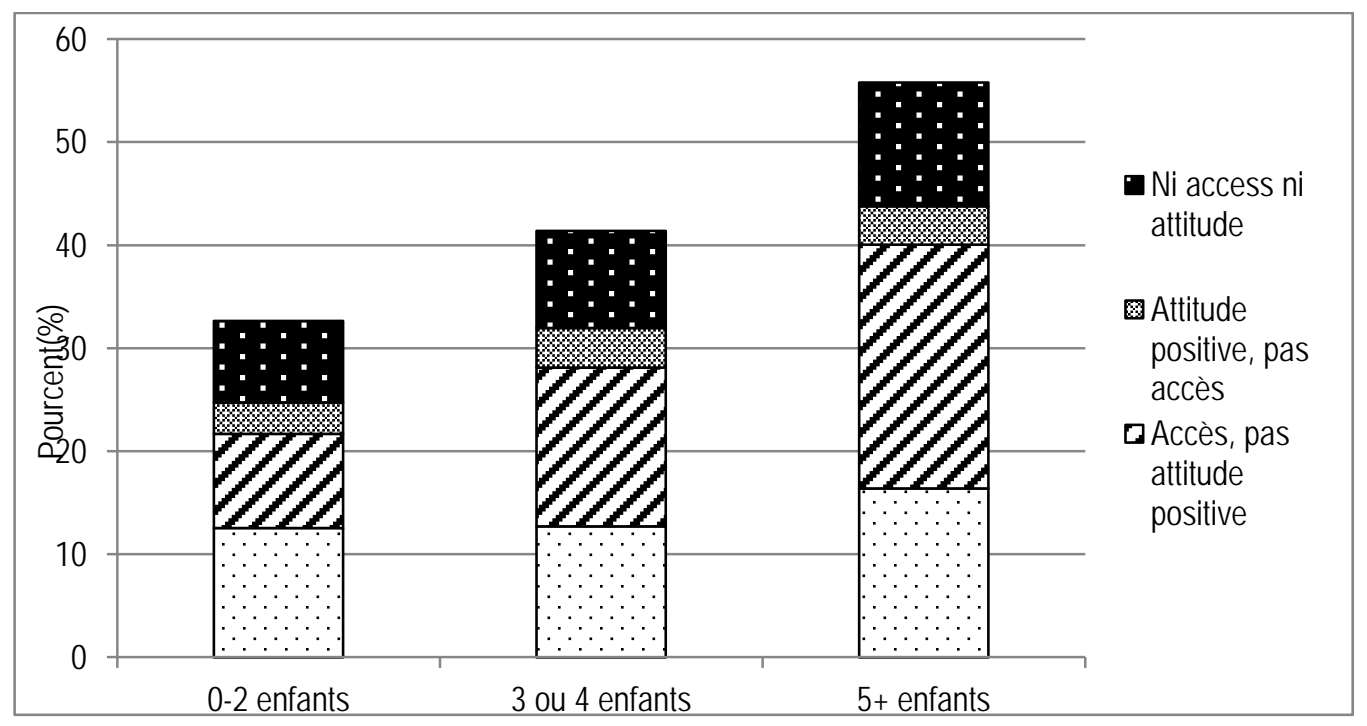

\section{Régressions logistiques multivariée et multinomiale}

L'analyse a été jusqu'ici essentiellement descriptive. Afin de pouvoir identifier de plus près les facteurs qui conduisent à un besoin non satisfait, la régression logistique a été appliqué et les résultats sont présentés dans le Tableau A.4. Les odds ratios ajustés indiquent l'association entre caractéristiques personnelles et le fait d'avoir un besoin non satisfait ou d'être une utilisatrice de méthode moderne, après contrôle des autres facteurs.

Les différences géographiques étaient grandes. Les femmes rurales étaient beaucoup plus susceptibles d'avoir un besoin non satisfait que les femmes urbaines. Les femmes habitant dans la région du sud-est étaient 1.9 fois plus susceptible de signaler un besoin non satisfait que les femmes habitant dans la région de l'ouest. Les femmes de la région centre avaient également un risque 1.6 fois plus élevé de besoin non satisfait que celles de la région de l'ouest, après contrôle des autres facteurs. L'inégalité dans l'instruction était flagrante. Les femmes ayant une instruction de niveau primaire ou plus avaient moitié moins de chance d'avoir un besoin non satisfait que les femmes n'ayant pas été à l'école. Les femmes dans la quintile la plus pauvre avaient un risque 1.7 plus élevé d'avoir un besoin non satisfait que les femmes dans la quintile moyenne. L'âge n'a pas été associé au besoin non satisfait mais la parité l'a été. Les femmes ayant moins de 3 enfants avaient nettement plus de besoins non satisfaits que celles ayant $3-4$ enfants. Le besoin non satisfait était également plus élevé parmi les femmes allaitantes.

Les résultats d'une analyse multivariée plus complexe qui utilise une régression logistique multinominale sont présentés dans le Tableau A.5. Les non-utilisatrices y sont divisées en cinq catégories basées sur nos quatre combinaisons d'accès et d'attitude, avec une cinquième catégorie pour celles qui veulent un enfant au cours des deux prochaines années et donc ont un besoin faible ou aucun besoin non satisfait et une raison convaincante de non-utilisation. Les ratios de risque relatif ajusté montrent les ratios de probabilité d'être dans chacune des cinq catégories de la non-utilisation relative à la probabilité d'être une utilisatrice de méthode moderne. 
Les ratios de risque relatifs montrent qu'en comparaison aux femmes dans les zones urbaines, celles dans les zones rurales étaient plus susceptibles de se trouver dans les quatre catégories de besoin non satisfait et de vouloir un enfant bientôt. Toutes, sauf une de ces différences étaient significatives au niveau de confiance de 95\%. Les conclusions indiquent que les femmes dans les zones rurales étaient plus susceptibles d'avoir un besoin non satisfait et que le manque d'accès et d'attitude d'acceptation sont des problèmes plus communs parmi elles que parmi les femmes urbaines.

Les effets les plus marqués dans le Tableau A.5 concernent l'instruction et la résidence géographique (résidence et région). Comparé au les femmes avec quelque instruction, les femmes sans instruction avaient nettement moins d'accès et, lorsqu'elles avaient l'accès, étaient plus susceptibles d'avoir une attitude défavorable.

Comparé aux femmes dans la région de l'ouest, les femmes dans toutes les régions sauf le sud étaient plus de 2.3 fois plus susceptibles d'avoir un besoin non satisfait sans accès ni attitude que d'être utilisatrices de contraception moderne. Les résultats suggèrent que les attitudes étaient plus favorables dans le sud qu'ailleurs; ces femmes étant nettement moins susceptibles d'appartenir à la catégorie de besoin non satisfait avec accès mais sans attitude positive. Les femmes du sud-est et, dans une moindre mesure, les femmes de la région centre, étaient plus susceptibles de vouloir un autre enfant très prochainement que celles des autres régions.

Le manque d'accès était plus évident parmi les femmes dans la quintile la plus pauvre. Comparées avec les femmes dans la quintile moyenne, elles avaient 3.0 fois plus de chance d'avoir un besoin non satisfait sans accès que d'être utilisatrices de contraceptif moderne. II n'existait pas de différence entre la chance d'appartenir au groupe avec accès et attitude comparé à être une utilisatrice de contraceptif moderne selon le niveau d'instruction et le quintiles de richesse. Comme discuté ci-dessous, les raisons de besoin non satisfait parmi les femmes ayant l'accès et une attitude positive inclus l'allaitement ou les rapports sexuels peu fréquents.

Le besoin non satisfait parmi les femmes allaitantes était élevé. Basé sur la régression multinominale, les raisons découlaient de l'attitude et de l'accès. De façon prévisible, les femmes allaitantes étaient beaucoup moins susceptibles que les autres femmes de vouloir un enfant tôt. Les femmes à faible parité avaient également un besoin non satisfait élevé mais ni l'attitude ni l'accès ne semblent être un problème particulier.

\section{Raisons de non-utilisation de la planification familiale}

En réponse à une question sur les raisons du besoin non satisfait, de nombreuses réponses étaient permises; environ $5 \%$ ont fourni plus d'une raison (Tableau A.6). Les raisons principales pour la nonutilisation de la planification familiale étaient l'opposition de la répondante (18.6\%) et les rapports peu fréquents (17.7\%) ; l'allaitement et les préoccupations de santé étaient également souvent mentionnés (Tableau 1).

Le Tableau 1 compare les quatre catégories de besoins non satisfaits, définis en termes d'accès et d'attitude avec les raisons auto-déclarées. Dans le tableau, les trois premières raisons sont relatives à l'attitude de résistance et les deux suivantes sont associées à des problèmes d'accès. Les autres raisons identifient d'autres problèmes. Les raisons varient parmi les quatre catégories. Parmi les femmes 
définies comme ayant l'accès et l'attitude, les rapports peu fréquents (22.8\%), l'allaitement (19.1\%) et les préoccupations liées à la santé (15.5\%) étaient les plus mentionnés, i.e. des raisons pas associées à l'accès ou à l'attitude. Comme attendu, dans le groupe avec l'attitude mais sans l'accès la raison la plus commune de non-utilisation était le manque de connaissance et l'opposition était mentionnée moins fréquemment.

Tableau 1: Raisons de non-utilisation de planification familiale par catégorie de besoin non satisfait ${ }^{3}$

\begin{tabular}{|c|c|c|c|c|c|}
\hline & $\begin{array}{l}\text { accès et } \\
\text { attitude }\end{array}$ & $\begin{array}{c}\text { accès, mais } \\
\text { pas } \\
\text { d'attitude }\end{array}$ & $\begin{array}{l}\text { attitude, } \\
\text { mais pas } \\
\text { d'accès }\end{array}$ & $\begin{array}{l}\text { ni accès ni } \\
\text { attitude }\end{array}$ & TOTAL \\
\hline $\begin{array}{l}\text { Opposition de la } \\
\text { répondante } \\
\text { Opposition du }\end{array}$ & 7.5 & 25.3 & 10.4 & 27.8 & 18.6 \\
\hline partenaire/autrui & 10.1 & 14.4 & 9.2 & 10.3 & 11.7 \\
\hline Religion & 1.0 & 4.4 & 1.8 & 5.2 & 3.2 \\
\hline Méconnaissance & 4.8 & 2.3 & 15.8 & 6.0 & 5.1 \\
\hline Accès/coût & 7.8 & 2.3 & 12.7 & 1.1 & 4.8 \\
\hline $\begin{array}{l}\text { Rapports peu } \\
\text { fréquents/non existants }\end{array}$ & 22.8 & 14.9 & 15.7 & 15.3 & 17.7 \\
\hline $\begin{array}{l}\text { Allaitement } \\
\text { Préoccupations de } \\
\text { santé/effets } \\
\text { secondaires/interfère }\end{array}$ & 19.1 & 14.5 & 17.4 & 15.6 & 16.5 \\
\hline avec le corps & 15.5 & 15.9 & 13.8 & 11.8 & 14.7 \\
\hline Fataliste & 0.4 & 1.2 & 0.8 & 0.7 & 0.8 \\
\hline Autres/ne sait pas & 12.3 & 4.8 & 3.5 & 5.5 & 7.3 \\
\hline $\mathrm{N}$ & 550 & 630 & 142 & 337 & 1660 \\
\hline
\end{tabular}

En revanche, parmi les deux groupes de femmes avec une attitude défavorable, l'opposition de la répondante était la raison la plus fréquemment mentionnée. L'opposition du partenaire était également la cinquième raison la plus citée. Les femmes n'ayant ni l'accès ni l'attitude avaient un profil semblable, mais la proportion citant l'opposition du partenaire était relativement plus petite et la méconnaissance était plus élevée.

3286 femmes ayant un besoin non satisfait n'avaient pas d'information sur les raisons de ne pas utiliser la planification familiale: 213 femmes n'ont pas été interrogées parce qu'elles ont donné des réponses non numériques à la question sur leur temps d'attente préféré et 73 femmes n'ont pas été interrogées car elles ne savaient pas encore si elles voulaient d'autres enfants. 
Figure 8: Raison de non-utilisation selon la zone géographique

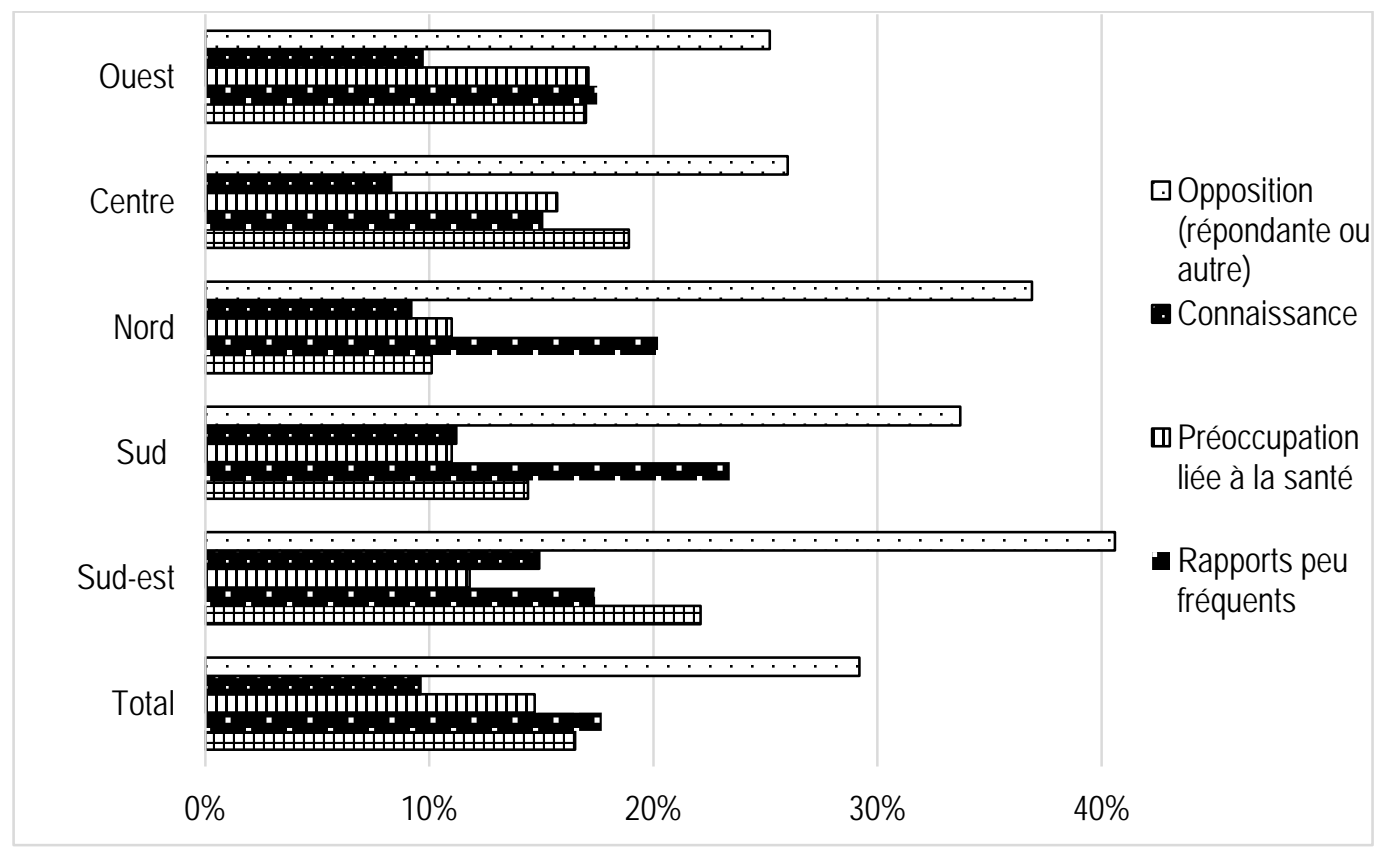

La Figure 8 montre les raisons principales pour non-utilisation selon les zones géographiques. Dans la zone sud-est, la proportion des femmes qui ont mentionné l'opposition de la répondante, du mari ou d'autres étaient particulièrement élevée (40.6\%), suivi par l'allaitement et les rapports peu fréquents. Dans les régions du nord et du sud, les rapports peu fréquents et l'opposition étaient également communs. Les zones de l'ouest et du centre avaient des profils semblables. En plus de l'opposition, les préoccupations liées à la santé, l'allaitement et les rapports peu fréquents ont été le plus mentionnés, et les problèmes liés à l'accès ou à la connaissance étaient moins fréquemment mentionnés.

Les variations dans les raisons auto-déclarées parmi d'autres strates de la population ont été explorées (données non présentées). L'opposition de la répondante variait d'une manière significative selon le niveau d'instruction, de richesse et l'état postpartum : les femmes moins instruites et les femmes ayant accouché il y a plus de 2 ans ont mentionné l'opposition en plus grande proportions. Les rapports peu fréquents variaient selon la résidence, l'instruction, la richesse et l'état postpartum. Ils ont été plus cités par les femmes les plus riches, les plus instruites et les urbaines. Les rapports peu fréquents ont été plus mentionnés par les femmes ayant accouché depuis plus de 2 ans que par celles qui ont accouché au cours des 2 années précédant l'enquête.

\section{Opposition à l'utilisation}

L'opposition de la répondante était une des raisons principales de non-utilisation au Sénégal. Cela découle peut-être des croyances personnelles ou de l'opposition du partenaire ou de la famille. La religion est rarement mentionnée. L'opposition par les maris ou autrui a été moins fréquemment citée que l'opposition de la répondante elle-même mais une distinction nette entre les deux est difficile à faire puisqu'il est probable que les points de vue des partenaires s'influencent. Une raison possible pourrait être une faible exposition au message de planification familiale. En effet, seulement $58 \%$ des femmes ont 
déclaré qu'elles ont déjà entendu ou lu des messages sur la planification familiale à la télé, à la radio ou dans un journal au court du mois précédent. Parmi celles exposées aux messages de planification familiale, $26.7 \%$ ont mentionné l'opposition comme motif de non-utilisation comparées à $31.1 \%$ pour celles qui n'ont pas été exposées aux messages. Bien que la différence n'était pas significative, les femmes ayant reçu le message de la planification familiale semblent avoir une attitude moins négative à l'égard la planification familiale.

La figure 9 présente les proportions de femmes qui avaient entendu les messages de planification familiale au cours du mois précédent. II existe des différences significatives parmi les strates de population. Moins d'un tiers des femmes rurales avaient entendu des messages comparées à presque $60 \%$ des femmes urbaines. L'exposition dans la zone ouest est deux fois plus élevée qu'ailleurs. Les inégalités selon le niveau d'instruction et de quintile de richesse étaient significatives. Seules $15.0 \%$ des femmes dans le quintile le plus pauvre avaient entendu des messages au cours du mois précédent.

Figure 9: Proportion de femmes qui avaient entendu des messages de planification familiale soit à la télé, à la radio ou dans un journal dans le mois précédent selon les strates de population

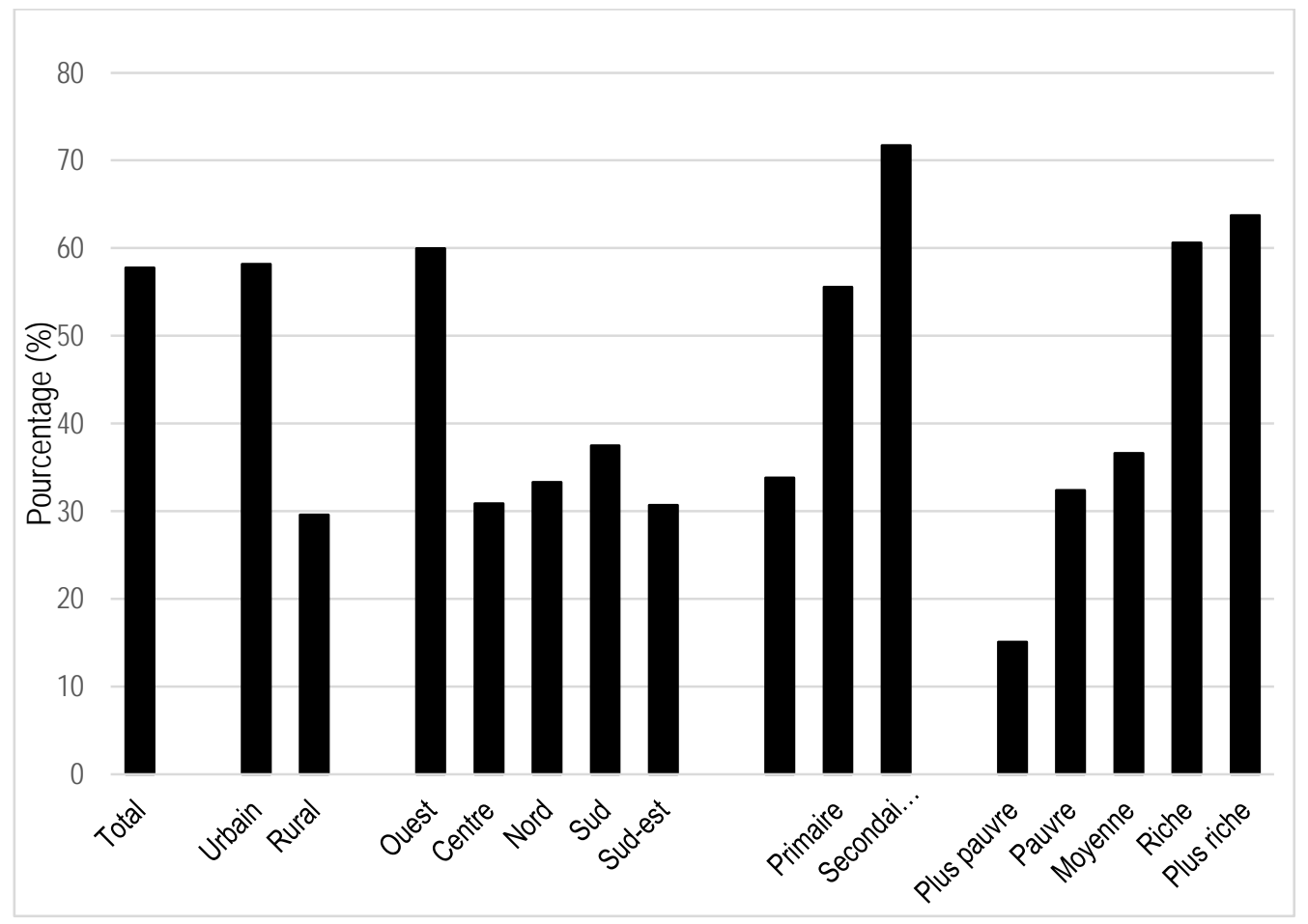

Rapports sexuels peu fréquents

Les rapports sexuels peu fréquents ont été mentionnés comme motif de non-utilisation. Pour servir de vérification partielle à cette réponse, les réponses à une question indépendante sur la récence des derniers rapports sexuels ont été examinées. Ceci ne mesure pas la fréquence moyenne des rapports sexuels au cours d'une année, c'est la seule information disponible dans l'EDS et elle peut être considérée comme un indicateur de fréquence des rapports sexuels. Les femmes citant des rapports peu 
fréquents étaient en effet moins susceptibles que les autres de déclarer avoir eu des rapports sexuels au cours des 4 semaines précédentes (Tableau 2). Néanmoins, un tiers ont déclaré avoir eu des rapports sexuels dans un passé récent même si la fréquence a pu être faible.

Table 2: Récence des derniers rapports sexuels selon que rapports sexuels peu fréquents aient été donné comme motif de non-utilisation

\begin{tabular}{|c|c|c|c|}
\hline \multirow{2}{*}{$\begin{array}{l}\text { Récence du dernier } \\
\text { rapport }\end{array}$} & \multicolumn{2}{|c|}{$\begin{array}{l}\text { Raison de non-utilisation: } \\
\text { rapports sexuels peu fréquent }\end{array}$} & \multirow[b]{2}{*}{ Tota } \\
\hline & Non (\%) & Oui (\%) & \\
\hline $\begin{array}{l}\text { Au cours des } 4 \\
\text { dernières semaines }\end{array}$ & 81.7 & 34.7 & 72.9 \\
\hline \multicolumn{4}{|c|}{ Au cours des 3 derniers } \\
\hline mois & 13.2 & 36.7 & 16.8 \\
\hline Il y a 4 mois ou plus & 5.0 & 28.2 & 8.6 \\
\hline $\begin{array}{l}\text { Avant le dernier } \\
\text { accouchement }\end{array}$ & 0.2 & 0.4 & 0.2 \\
\hline Total & 100.0 & 100.0 & 100 \\
\hline
\end{tabular}

Afin de tester l'hypothèse que les rapports sexuels peu fréquents sont une alternative à la contraception, nous avons examiné par régression logistique les corolaires de l'abstinence sexuel dans le mois précédent (Tableau A.7). Après ajustement pour l'utilisation de la contraception, le statut résidentiel, le type de mariage (polygame ou monogame), l'allaitement, la parité et l'âge, des associations significatives ont été trouvées entre le désir pour, et le moment pour, avoir un autre enfant et l'abstinence. Le statut résidentiel était un prédicateur fort de ne pas avoir eu de rapports sexuels dans les 4 dernières semaines. En répondant à la question «votre mari/partenaire habite-t-il avec vous actuellement ou loge-t-il ailleurs ? » les femmes déclarant que leur mari logeait ailleurs étaient 12.5 fois plus susceptibles que les autres de s'être abstenues des rapports sexuels au cours des derniers 28 jours. Parmi les femmes exposées mariées ou en cohabitation, 35\% ont déclaré que leur mari logeait ailleurs au moment de l'enquête. Néanmoins, après ajustement pour le statut résidentiel, l'utilisation de contraception, le type de mariage, l'instruction, la parité, la situation de postpartum et d'autres facteurs, des associations significatives ont été trouvées entre l'abstinence et le désir d'arrêter d'avoir des enfants. Les femmes qui ne voulaient plus d'enfants étaient 1.5 fois plus susceptibles de s'être abstenues au cours des 4 dernières semaines. En outre, les non utilisatrices de contraceptif étaient 1.8 fois plus susceptibles de déclarer l'abstinence que les utilisatrices. Les femmes ayant moins de 3 enfants étaient nettement plus susceptibles de ne déclarer aucun rapport. II est possible que les couples ayant $0-2$ enfants font preuve une résistance plus forte à l'utilisation d'une méthode moderne car ils n'ont pas encore eu tous les enfants qu'ils souhaitent ou le nombre d'enfants attendus par la société. Tandis que l'arrangement résidentiel est un déterminant majeur, ces résultats vont dans le sens de l'opinion que la réduction de la fréquence des rapports est utilisée au Sénégal en tant qu'alternative aux méthodes de contraceptions. 


\section{Discussion et Implications}

Le besoin non satisfait en contraceptif est élevé au Sénégal. Parmi les femmes mariées ou en cohabitation exposées au risque de tomber enceintes cohabitation au moment de l'enquête, mais qui veulent retarder une grossesse pour au moins deux ans, $41 \%$ ont déclaré qu'elles n'utilisaient pas de méthode moderne, une estimation des besoins non satisfaits plus élevée que celle donnée dans le Rapport principal de l'EDS Sénégal 2010-11 qui incluait les femmes non exposées.

L'impression dominante de cette analyse est peut-être typique d'un pays africain à un stade précoce du changement reproductif. La taille moyenne de famille désirée reste élevée à 5.2 enfants, ce qui correspond au taux de fécondité actuel. Dans l'échantillon de 4663 femmes exposées, plus d'un tiers (36\%) voulaient un autre enfant dans les 2 ans et $79 \%$ voulaient un enfant bientôt ou plus tard. La plupart des besoins non satisfaits découle du désir d'espacer ou de retarder la prochaine grossesse. L'abstinence postpartum est courte (3.5 mois). Les contraintes traditionnelles sur la conception sont insuffisantes. Les durées moyennes d'allaitement et l'aménorrhée sont beaucoup plus longues, à 21 et 12 mois respectivement (Agence Nationale de la Statistique et de la Démographie (ANSD) [Sénégal] and ICF International 2012), mais restent insuffisantes pour atteindre les intervalles souhaités entre naissances. En outre, la durée de la protection lactationelle a diminué de plusieurs mois au cours des deux dernières décennies. En 2005, la durée moyenne de l'intervalle préférée était estimée à 36 mois comparée à un intervalle moyen actuel de 32 mois et $56 \%$ des femmes ont vécu un intervalle plus court que celui qu'elles auraient préféré (Rutstein 2011). En 2010-11, 24\% des naissances récentes ont été signalées comme accidentelles mais 20\% étaient survenues à un mauvais moment et seulement 4\% étaient non désirées. (Agence Nationale de la Statistique et de la Démographie (ANSD) [Sénégal] and ICF International 2012). Le besoin de contraception pour espacer les naissances est clair.

L'un des principaux objectifs de cette analyse est de déterminer l'importance du manque d'accès et de l'attitude de résistance comme origine du besoin non satisfait. II s'est avéré que l'attitude de résistance était plus généralisée que le manque d'accès. Parmi les femmes ayant un besoin non satisfait, $61 \%$ n'avaient pas l'intention d'utiliser à l'avenir et étaient donc classées comme ayant une attitude défavorable, tandis que $31 \%$ ne connaissaient pas les pilules et les injectables et/ou ne connaissaient pas une source d'approvisionnement et étaient donc classées comme n'ayant pas accès. Une attitude positive était associée à l'accès; environ la moitié de celles ayant accès avaient une attitude favorable par rapport à moins d'un quart de celles sans accès. La résistance à la contraception découle donc en partie du manque de connaissance. Peu de femmes ayant un besoin non satisfait ont déjà essayé une méthode moderne.

L'attitude répandue de résistance est confirmée par les réponses données à une question directe sur les motifs de non-utilisation. La raison la plus citée, donnée par 19\%, était l'opposition de la répondante à la contraception et $12 \%$ ont cité l'opposition du mari ou d'autrui. L'opposition de la répondante a été citée beaucoup plus souvent par les femmes n'ayant aucune intention d'utiliser dans l'avenir que par celles ayant l'intention d'utiliser, mais l'opposition d'autrui était une réponse tout aussi commune dans les deux groupes. Les résultats ne permettent pas d'évaluer la force de la résistance mais il est intéressant de noter que l'inquiétude initiale, et les soupçons, par rapport à, l'idée de la contraception et des méthodes spécifiques est normale dans les pays ayant des niveaux bas d'instruction des adultes. Cela peut prendre du temps et des efforts de communication pour que la contraception devienne acceptée et pas sujet de 
controverse dans la vie quotidienne des personnes mariées, mais, jugeant par l'expérience d'autres pays pauvres au cours des 50 dernières années, le changement est inévitable.

Un motif commun de non-utilisation est les rapports sexuels peu fréquents. La proportion donnant cette réponse ne varie pas énormément parmi les quatre catégories principales de besoin non satisfait, définie en termes d'attitude et accès, même si c'est le motif le plus commun donné par les femmes ayant accès et une attitude positive. Ce motif apparaît en haut de la liste des motifs dans le questionnaire ce qui pourrait faire croire que son emplacement influence les réponses. Cependant, la comparaison avec une question indépendante sur la récence des derniers rapports sexuels suggère que les rapports peu fréquents ne sont pas simplement un artéfact dans la conception du questionnaire ou une réponse trompeuse. Seulement $32.5 \%$ des femmes donnant cette raison de non-utilisation ont déclaré avoir eu des rapports sexuels au cours du dernier mois contre $81 \%$ pour les autres femmes. L'analyse multivariée des corolaires de l'abstinence sexuelle récente a montré que l'abstinence était fortement associée avec le fait que leurs maris habitaient avec elles dans le même ménage au moment de l'enquête. Dans le modèle de la famille sénégalaise typique, un couple n'habite pas forcément ensemble dans le même ménage. Selon l'estimation de Findley, entre $43 \%$ et $68 \%$ des couples vivent séparément pendant quelques années à un moment donné de leurs vies au Sénégal (Findley 1997). Un nombre croissant de femmes au Sénégal ont déclaré que leur mari vivait ailleurs au moment de l'enquête. Dans l'EDS Sénégal 2010-11, 35\% des femmes en union exposées ne vivaient pas avec leur mari ou partenaire. Néanmoins, même après ajustement pour la cohabitation, l'abstinence était plus commune chez celles qui ne souhaitaient plus avoir d'enfants que chez celles qui voulaient un autre enfant prochainement, et également plus élevée parmi les non utilisatrices que les utilisatrices de contraceptif. Ces résultats suggèrent que l'abstinence, ou la réduction de la fréquence des rapports sexuels, peut être utilisée comme alternatif partiel à la contraception. Ce comportement ne semble pas prendre la forme d'une abstinence périodique; cette méthode a été explicitement introduite aux participantes comme étant une méthode possible dans cette partie du questionnaire portant sur la connaissance de la contraception, mais très peu de femmes ont déclaré l'utiliser. Cependant, l'abstinence, autre que pour éviter les rapports à certains moments durant le cycle menstruel, n'a pas été identifiée comme méthode dans le questionnaire et donc resterait non déclarée. Dans le premier EDS du 1986, l'abstinence avait été acceptée comme méthode pour éviter la grossesse et avait été la méthode la plus souvent citée à ce moment-là. Cependant, l'abstinence n'a pas été une méthode pré-spécifiée dans le Questionnaire depuis le deuxième tour de l'EDS, et uniquement l'abstinence périodique et le retrait sont spécifiés. De ce fait, avec les données EDS, aucun progrès ne peut être fait pour élucider le rôle possible de l'abstinence dans le mariage en tant que moyen de réduire le risque de grossesse. Des recherches ethnographiques pourraient porter fruit.

Le besoin non satisfait était très élevé chez les femmes allaitantes. La raison principale était que les femmes allaitant un enfant de deux ans ou moins étaient nettement moins susceptibles de vouloir un autre enfant prochainement que les femmes ayant sevré leur enfant mais qui n'étaient pas plus susceptibles d'utiliser une méthode contraceptive. L'allaitement était un motif commun de non-utilisation dans les quatre catégories de besoins non satisfaits. Évidemment, les inquiétudes existent sur la compatibilité de la contraception et l'allaitement.

Les différences géographiques et socio-économiques dans l'utilisation actuelle d'une méthode moderne étaient très prononcées. Par exemple, plus de $30 \%$ des femmes urbaines et instruites étaient utilisatrices contre $12 \%$ des femmes rurales et $16 \%$ de celles sans instruction. La prévalence contraceptive était 32\% dans la région de l'Ouest mais de seulement $10 \%$ dans la partie Sud-est du pays. Les différences de besoins non satisfaits étaient également significatifs mais pas aussi grandes que celles pour l'utilisation car le désir d'un enfant dans un avenir proche a tendance à être plus prévalent parmi les groupes où 
l'utilisation est faible. II est également vrai que le besoin non satisfait et les attitudes défavorables sont communs dans toutes les strates de population examinées. Même dans la petite élite de femmes urbaines ayant une instruction secondaire, 32\% avaient un besoin non satisfait et, parmi celles-ci, $42 \%$ n'avaient aucune intention d'utiliser dans l'avenir.

La mise en œuvre historiquement faible des programmes de planification familiale au Sénégal est évidente par le fait qu'en 2010-11, presqu'un tiers des femmes mariées ou en cohabitation ne connaissaient pas les deux méthodes principales de contraception et/ou n'étaient pas au courant d'une source d'approvisionnement. Ce manque d'accès est particulièrement commun dans les régions Centre, Nord et Sud-est du pays. De ce fait, une première priorité politique et une qui est relativement facile à réaliser est d'accroître la connaissance à travers les médias de masse et des activités locales de sensibilisation. Un objectif aussi important, mais plus difficile est d'améliorer le climat de l'opinion sur la contraception. Dans les zones urbaines, un progrès significatif a été fait : 32\% utilisent une méthode moderne et $17 \%$ ont l'intention de le faire à l'avenir. Dans les zones rurales, l'utilisation et les attitudes positives sont à un niveau beaucoup plus bas. Un plaidoyer actif pour la planification familiale par les leaders politiques, religieux et traditionnels aussi bien au niveau national que local, pourrait être nécessaire pour obtenir une légitimation sociale. De plus, des efforts pour prendre en compte le besoin non satisfait élevé des femmes allaitantes constituent une priorité plus focalisée.

Le gouvernement sénégalais a récemment repositionné la politique de la planification familiale et s'est engagé à doubler le budget total pour la gestion des programmes de planification familiale. Les deux recommandations ci-dessus sont clairement notées comme deux des six piliers du Plan d'Actions National de Planification Familiale 2011-2015. La politique forte est maintenant en place et attend sa mise en œuvre efficace. 


\section{Références}

Agence Nationale de la Statistique et de la Démographie (ANSD) [Sénégal]and ICF International. 2012. Enquête Démographique et de Santé à Indicateurs Multiples au Sénégal (EDS-MICS) 2010-2011. Calverton, Maryland,USA: ANSD and ICF International.

Bradley, S.E.K., T.N. Croft, J.D. Fishel, and C.F. Westoff. 2012. Revising unmet need for family planning. DHS Analytical Studies. No.25. Calverton, MD: ICF Macro. Retrieved from http:www.measuredhs.com/pubs/pdf/AS25/AS25[12June2012].pdf.

Cleland, J.G., R.P. Ndugwa, and E.M. Zulu. 2011. Family planning in sub-Saharan Africa: progress or stagnation? Bulletin of the World Health Organization 89(2):137-143.

Coale, A.J. 1973. The demographic transition reconsidered. (Eds). International Union for the Scientific Study of Population, Liege, Belgium.

Findley, S. 1997. Migration and family interactions in Africa. Pp. 109-138 in A. Adepoju (Eds). Family, Population and Development in Africa, London: Zed Books.

Lesthaeghe, R.and C. Vanderhoeft. 2001. Ready, willing, and able: a conceptualization of transitions to new behavioral forms. Pp. 240-264. in J.B. Casterline (Eds). Diffusion processes and fertility transition: selected perspectives, Washington, D.C.: National Academy Press. 


\section{Annexe}

Figure A.1: Carte du Sénégal

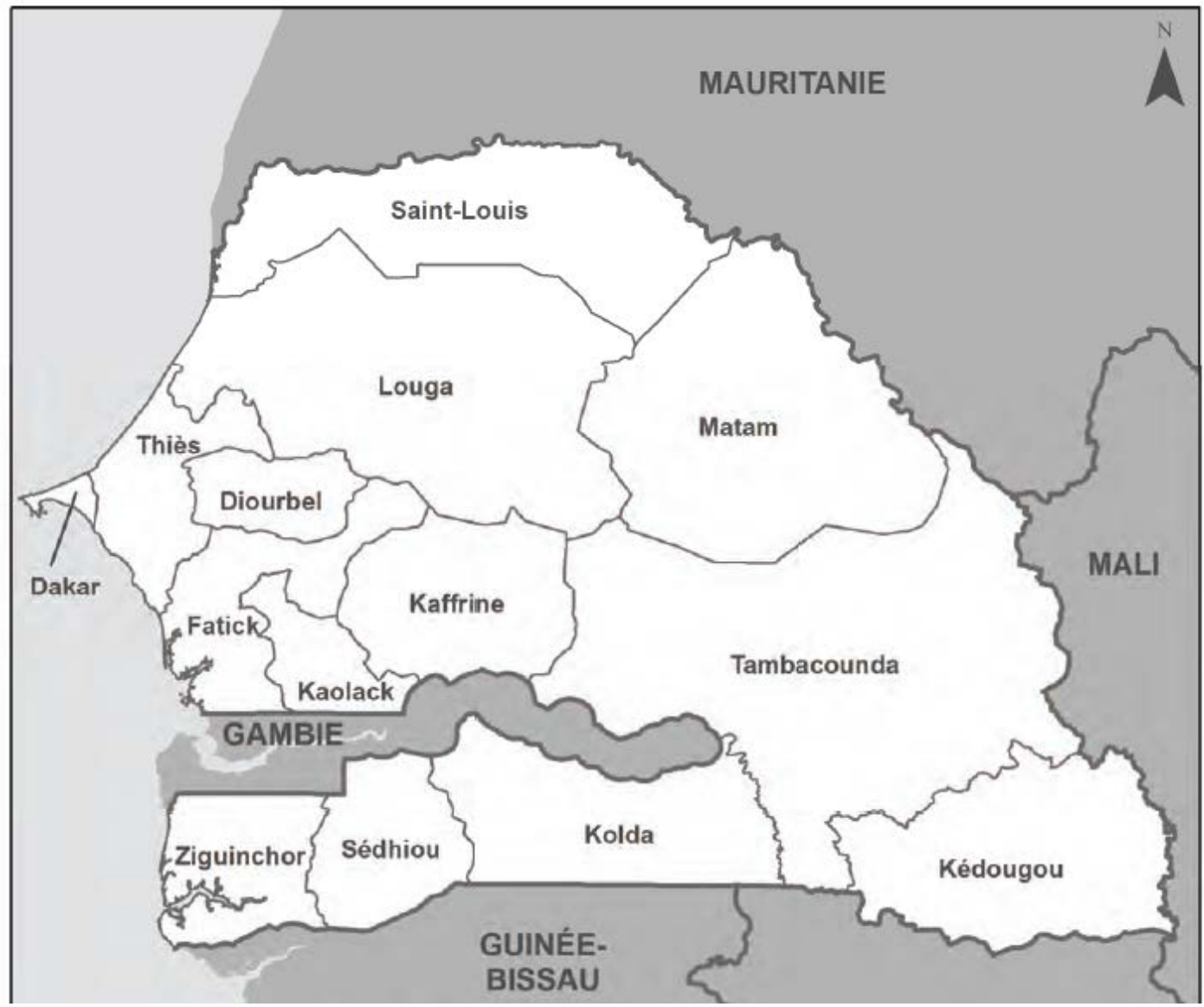

Ouest: Régions de Dakar et Thiès

Centre: Régions de Diourbel, Fatick, Kaolack et Kaffrine

Nord: Régions de Saint-Louis, Louga et Matam

Sud: Régions de Ziguinchor, Sédhiou et Kolda

Sud-est: Régions de Tambacounda et Kédougou

Source: Rapport final EDS 2010-2011 Sénégal. 
Table A.1: Définition des indicateurs utilisés pour l'analyse (Tous les indicateurs sont limités aux femmes fécondes/non enceintes qui ne s'abstiennent pas et qui sont actuellement mariées ou en cohabitation.)

\begin{tabular}{|c|c|c|}
\hline & Indicateur & Définition \\
\hline 1 & $\begin{array}{l}\text { Besoin non satisfait en PF (accès \& } \\
\text { attitude) }\end{array}$ & $\begin{array}{l}\text { \% de toutes les femmes qui ont l'accès (connaissance de la } \\
\text { pilule et des injectables et d'une source } \\
\text { d'approvisionnement) et l'attitude (intention d'utiliser dans } \\
\text { l'avenir) envers l'utilisation de la PF et veulent retarder le } \\
\text { prochain enfant pour au moins 2+ ans, mais N'utilisent PAS } \\
\text { de méthode moderne parmi toutes les femmes }\end{array}$ \\
\hline 2 & $\begin{array}{l}\text { Besoin non satisfait en PF (accès } \\
\text { mais pas d'attitude) }\end{array}$ & $\begin{array}{l}\text { \% de toutes les femmes qui ont l'accès, mais pas l'attitude } \\
\text { envers l'utilisation de la PF et veulent retarder le prochain } \\
\text { enfant pour au moins } 2+\text { ans, mais N'utilisent PAS de } \\
\text { méthode moderne parmi toutes les femmes }\end{array}$ \\
\hline 3 & $\begin{array}{l}\text { Besoin non satisfait en PF (attitude, } \\
\text { mais pas d'accès) }\end{array}$ & $\begin{array}{l}\text { \% de toutes les femmes qui ont l'attitude, mais pas l'accès à } \\
\text { l'utilisation de la PF et veulent retarder le prochain enfant } \\
\text { pour au moins } 2+\text { ans, mais N'utilisent PAS de méthode } \\
\text { moderne parmi toutes les femmes }\end{array}$ \\
\hline 4 & $\begin{array}{l}\text { Besoin non satisfait en PF (ni accès } \\
\text { ni attitude) }\end{array}$ & $\begin{array}{l}\text { \% de toutes les femmes qui n'ont ni l'accès ni l'attitude à } \\
\text { l'utilisation de la PF et veulent retarder le prochain enfant } \\
\text { pour au moins } 2+\text { ans, mais N'utilisent PAS de méthode } \\
\text { moderne parmi toutes les femmes }\end{array}$ \\
\hline 5 & $\begin{array}{l}\text { Vouloir un autre enfant et utiliser une } \\
\text { méthode moderne }\end{array}$ & $\begin{array}{l}\% \text { de toutes les femmes qui veulent un autre enfant qui } \\
\text { utilisent une méthode moderne parmi toutes les femmes }\end{array}$ \\
\hline 6 & $\begin{array}{l}\text { Ne pas vouloir d'enfant et utiliser } \\
\text { une méthode moderne pour limiter }\end{array}$ & $\begin{array}{l}\% \text { de toutes les femmes qui ne veulent plus d'enfant et } \\
\text { utilisent une méthode moderne parmi toutes les femmes }\end{array}$ \\
\hline 7 & $\begin{array}{l}\text { Désirer une naissance au cours des } \\
2 \text { prochaines années }\end{array}$ & $\begin{array}{l}\text { \% de toutes les femmes qui veulent un autre enfant au cours } \\
\text { des } 2 \text { prochaines années parmi toutes les femmes (excluant } \\
\text { les utilisatrices actuelles de contraception moderne) }\end{array}$ \\
\hline
\end{tabular}


Table A.2: Répartitions des femmes, EDS Sénégal 2010-11

\begin{tabular}{|c|c|c|c|}
\hline & & N pondéré & $\%$ \\
\hline \multirow{6}{*}{ 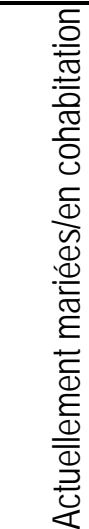 } & $\begin{array}{l}\text { Exposée au risque de tomber enceinte au moment de } \\
\text { l'enquête }\end{array}$ & 4,663 & 29.7 \\
\hline & $\begin{array}{l}\text { Féconde \& pas en abstinence postpartum \& ayant un besoin } \\
\text { non satisfait, mais données sur la connaissance d'une } \\
\text { source d'approvisionnement et l'intention non collectées ou } \\
\text { manquantes }^{\text {b }}\end{array}$ & 76 & 0.48 \\
\hline & Féconde \& en abstinence postpartum & 400 & 2.55 \\
\hline & Enceinte & 1159 & 7.39 \\
\hline & En aménorrhée & 2,242 & 14.3 \\
\hline & Stérile, ménopausée & 1,807 & 11.5 \\
\hline \multicolumn{2}{|c|}{ Jamais mariée/fût mariée } & 5,341 & 34.0 \\
\hline \multicolumn{2}{|l|}{ Total } & 15,688 & 100.0 \\
\hline
\end{tabular}


Table A.3: Répartition du besoin non satisfait en planification familiale, de l'utilisation de méthodes modernes et du désir de grossesse dans les 2 prochaines années

\begin{tabular}{|c|c|c|c|c|c|c|c|c|c|c|}
\hline & \multicolumn{2}{|c|}{ Total } & \multicolumn{2}{|c|}{ Résidence } & \multicolumn{5}{|c|}{ Zone géographique } \\
\hline & & $\begin{array}{c}\text { Pondéré } \\
\mathrm{N}\end{array}$ & $\%$ & \multirow[t]{2}{*}{ urbain } & rural & Ouest & Centre & Nord & Sud & Sud-est \\
\hline \multicolumn{10}{|c|}{ Pourcentages } & \\
\hline \multirow{4}{*}{ 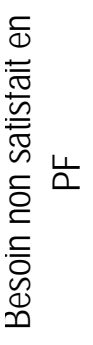 } & accès \&attitude & 642 & 13.8 & 15.3 & 12.3 & 14.8 & 13.6 & 11.7 & 14.7 & 12.3 \\
\hline & $\begin{array}{l}\text { accès, mais pas } \\
\text { attitude }\end{array}$ & 700 & 15.0 & 14.3 & 15.6 & 15.5 & 14.7 & 17.6 & 10.2 & 12.5 \\
\hline & $\begin{array}{l}\text { attitude, mais } \\
\text { pas accès }\end{array}$ & 160 & 3.4 & 2.6 & 4.3 & 2.2 & 5.1 & 3.0 & 3.6 & 5.4 \\
\hline & $\begin{array}{l}\text { ni accès ni } \\
\text { attitude }\end{array}$ & 444 & 9.5 & 5.8 & 13.0 & 5.0 & 12.6 & 14.6 & 6.2 & 16.3 \\
\hline \multirow{3}{*}{\multicolumn{2}{|c|}{$\begin{array}{l}\text { utilisation pour espacer } \\
\text { utilisation pour limiter } \\
\text { désir enfant en }<2 \text { ans }\end{array}$}} & 671 & 14.4 & 21.8 & 7.4 & 21.3 & 8.1 & 11.5 & 13.8 & 6.9 \\
\hline & & 360 & 7.7 & 10.6 & 5.0 & 11.0 & 5.2 & 5.1 & 9.0 & 3.1 \\
\hline & & 1686 & 36.2 & 29.5 & 42.4 & 30.3 & 40.7 & 36.5 & 42.6 & 43.6 \\
\hline \multicolumn{2}{|c|}{ Total } & 4663 & 100.0 & 100.0 & 100.0 & 100.0 & 100.0 & 100.0 & 100.0 & 100.0 \\
\hline \multicolumn{2}{|c|}{ Besoin non satisfait total } & & 41.7 & 38.0 & 45.2 & 37.5 & 45.9 & 46.9 & 34.6 & 46.5 \\
\hline \multicolumn{2}{|c|}{ valeur $-p$ pour test $x^{2}$} & 1946 & & & $<0.001$ & & & & & $<0.001$ \\
\hline
\end{tabular}

\begin{tabular}{|c|c|c|c|c|c|c|c|c|c|c|}
\hline \multicolumn{11}{|c|}{ N Pondéré } \\
\hline \multirow{4}{*}{ 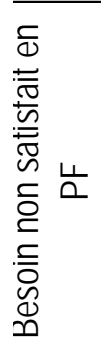 } & accès \&attitude & 642 & & 347 & 295 & 275 & 179 & 94 & 62 & 33 \\
\hline & $\begin{array}{l}\text { accès, mais pas } \\
\text { d'attitude }\end{array}$ & 700 & & 324 & 376 & 288 & 195 & 141 & 43 & 33 \\
\hline & $\begin{array}{l}\text { attitude, mais } \\
\text { pas d'accès }\end{array}$ & 160 & & 58 & 103 & 40 & 67 & 24 & 15 & 14 \\
\hline & $\begin{array}{l}\text { ni accès ni } \\
\text { attitude }\end{array}$ & 444 & & 131 & 313 & 92 & 166 & 117 & 26 & 43 \\
\hline \multirow{3}{*}{\multicolumn{2}{|c|}{$\begin{array}{l}\text { utilisation pour espacer } \\
\text { utilisation pour limiter } \\
\text { désir enfant en }<2 \text { ans }\end{array}$}} & 671 & & 494 & 178 & 396 & 107 & 92 & 58 & 18 \\
\hline & & 360 & & 241 & 119 & 204 & 69 & 41 & 38 & 8 \\
\hline & & 1686 & & 667 & 1019 & 562 & 537 & 292 & 179 & 116 \\
\hline \multicolumn{2}{|c|}{ Total } & 4663 & 0.41 & 2261 & 2402 & 1857 & 1320 & 801 & 420 & 265 \\
\hline
\end{tabular}




\begin{tabular}{|c|c|c|c|c|c|c|c|c|c|c|c|}
\hline & & \multicolumn{2}{|c|}{ Âge de la mère } & \multicolumn{3}{|c|}{ Instruction de la mère } & \multicolumn{5}{|c|}{ Richesse du ménage } \\
\hline & & $<30$ ans & $30+$ ans & $\begin{array}{c}\text { sans } \\
\text { instruction }\end{array}$ & primaire & secondaire+ & $\begin{array}{l}\text { le plus } \\
\text { pauvre }\end{array}$ & pauvre & milieu & riche & le plus riche \\
\hline \multicolumn{12}{|c|}{ Pourcentages } \\
\hline \multirow{4}{*}{ 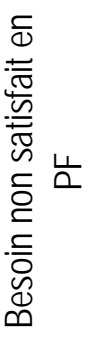 } & accès \&attitude & 13.2 & 14.3 & 12.3 & 16.3 & 16.4 & 11.6 & 12.9 & 13.3 & 14.1 & 15.9 \\
\hline & $\begin{array}{l}\text { accès, mais pas } \\
\text { d'attitude }\end{array}$ & 11.0 & 19.0 & 17.3 & 10.7 & 12.0 & 11.0 & 18.3 & 20.5 & 14.4 & 11.7 \\
\hline & $\begin{array}{l}\text { attitude, mais } \\
\text { pas d'accès }\end{array}$ & 4.1 & 2.8 & 4.4 & 2.1 & 1.2 & 7.1 & 3.9 & 2.7 & 3.3 & 1.4 \\
\hline & $\begin{array}{l}\text { ni accès ni } \\
\text { attitude }\end{array}$ & 10.4 & 8.6 & 11.9 & 7.0 & 2.9 & 19.0 & 11.3 & 9.3 & 7.3 & 4.2 \\
\hline \multirow{3}{*}{\multicolumn{2}{|c|}{$\begin{array}{l}\text { utilisation pour espacer } \\
\text { utilisation pour limiter } \\
\text { désir enfant en }<2 \text { ans }\end{array}$}} & 15.2 & 13.6 & 9.4 & 20.4 & 27.2 & 4.0 & 8.3 & 12.6 & 18.2 & 23.3 \\
\hline & & 0.9 & 14.5 & 6.8 & 10.0 & 8.1 & 4.9 & 5.7 & 8.3 & 8.7 & 9.6 \\
\hline & & 45.2 & 27.1 & 38.0 & 33.4 & 32.3 & 42.4 & 39.6 & 33.3 & 34.0 & 33.9 \\
\hline \multicolumn{2}{|c|}{ Total } & 100.0 & 100.0 & 100.0 & 100.0 & 100.0 & 100.0 & 100.0 & 100.0 & 100.0 & 100.0 \\
\hline \multirow{2}{*}{\multicolumn{2}{|c|}{$\begin{array}{l}\text { Besoin non satisfait total } \\
\text { p-value pour test du } x^{2}\end{array}$}} & 38.7 & 44.8 & 45.8 & 36.1 & 32.5 & 48.7 & 46.4 & 45.8 & 39.1 & 33.2 \\
\hline & & & $<0.001$ & & & $<0.001$ & & & & & $<0.001$ \\
\hline \multicolumn{12}{|c|}{ N Pondéré } \\
\hline \multirow{4}{*}{ 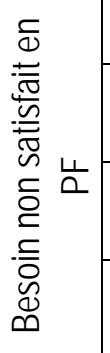 } & accès \&attitude & 308 & 334 & 359 & 182 & 102 & 89 & 102 & 117 & 151 & 184 \\
\hline & $\begin{array}{l}\text { accès, mais pas } \\
\text { attitude }\end{array}$ & 257 & 443 & 506 & 120 & 74 & 85 & 145 & 181 & 154 & 136 \\
\hline & $\begin{array}{l}\text { attitude, mais } \\
\text { pas accès }\end{array}$ & 96 & 65 & 129 & 24 & 8 & 54 & 31 & 24 & 35 & 16 \\
\hline & $\begin{array}{l}\text { ni accès ni } \\
\text { attitude }\end{array}$ & 243 & 201 & 348 & 78 & 18 & 146 & 89 & 82 & 79 & 49 \\
\hline \multirow{3}{*}{\multicolumn{2}{|c|}{$\begin{array}{l}\text { utilisation pour espacer } \\
\text { utilisation pour limiter } \\
\text { désir enfant dans }<2 \text { ans }\end{array}$}} & 354 & 318 & 275 & 229 & 168 & 31 & 66 & 111 & 195 & 269 \\
\hline & & 22 & 338 & 198 & 112 & 50 & 37 & 45 & 73 & 93 & 111 \\
\hline & & 1056 & 630 & 1113 & 374 & 199 & 325 & 312 & 293 & 365 & 391 \\
\hline \multicolumn{2}{|c|}{ Total } & 2335 & 2328 & 2927 & 1118 & 618 & 766 & 789 & 881 & 1072 & 1156 \\
\hline
\end{tabular}




\begin{tabular}{|c|c|c|c|c|c|c|c|c|c|c|}
\hline & & \multicolumn{4}{|c|}{ Richesse + Résidence } & \multicolumn{5}{|c|}{ Instruction + résidence } \\
\hline & & $\begin{array}{l}\text { urbain/ } \\
\text { pauvre }\end{array}$ & $\begin{array}{l}\text { urbain/ } \\
\text { riche }\end{array}$ & $\begin{array}{c}\text { rural/ } \\
\text { pauvre }\end{array}$ & rural/riche & $\begin{array}{c}\text { urbain/ } \\
\text { sans } \\
\text { instruction }\end{array}$ & $\begin{array}{l}\text { urbain/ } \\
\text { primaire }\end{array}$ & $\begin{array}{c}\text { urbain/ } \\
\text { secondaire+ }\end{array}$ & $\begin{array}{c}\text { rurall } \\
\text { sans } \\
\text { instruction }\end{array}$ & $\begin{array}{c}\text { rurall } \\
\text { primaire+ }\end{array}$ \\
\hline \multicolumn{11}{|c|}{ Pourcentages } \\
\hline \multirow{4}{*}{ 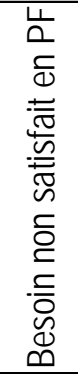 } & accès \&attitude & 12.6 & 16.1 & 12.5 & 12.0 & 13.2 & 17.0 & 17.0 & 11.8 & 14.4 \\
\hline & $\begin{array}{l}\text { accès, mais pas } \\
\text { attitude }\end{array}$ & 22.1 & 12.3 & 13.4 & 18.9 & 19.3 & 9.8 & 11.8 & 16.3 & 12.9 \\
\hline & $\begin{array}{l}\text { attitude, mais pas } \\
\text { accès }\end{array}$ & 4.3 & 2.1 & 5.4 & 2.7 & 4.3 & 1.3 & 1.2 & 4.5 & 3.4 \\
\hline & $\begin{array}{l}\text { ni accès ni } \\
\text { attitude }\end{array}$ & 9.0 & 5.0 & 15.6 & 9.3 & 6.8 & 7.2 & 2.0 & 14.4 & 6.8 \\
\hline \multirow{3}{*}{\multicolumn{2}{|c|}{$\begin{array}{l}\text { utilisation pour espacer } \\
\text { utilisation pour limiter } \\
\text { désir enfant dans }<2 \text { ans }\end{array}$}} & 15.2 & 23.6 & 5.8 & 9.7 & 17.2 & 22.6 & 29.3 & 5.5 & 15.6 \\
\hline & & 10.3 & 10.7 & 5.3 & 4.5 & 9.8 & 12.5 & 9.5 & 5.3 & 3.7 \\
\hline & & 26.6 & 30.3 & 42.1 & 42.9 & 29.5 & 29.7 & 29.3 & 42.2 & 43.3 \\
\hline \multicolumn{2}{|c|}{ Total } & 100.0 & 100.0 & 100.0 & 100.0 & 100.0 & 100.0 & 100.0 & 100.0 & 100.0 \\
\hline \multicolumn{2}{|c|}{ Besoin non satisfait total } & 47.9 & 35.4 & 46.9 & 42.9 & 43.5 & 35.2 & 32.0 & 47.0 & 37.5 \\
\hline \multicolumn{2}{|c|}{$\mathrm{p}$-value pour test du $x^{2}$} & & & & $<0.001$ & & & & & $<0.001$ \\
\hline
\end{tabular}

\begin{tabular}{|c|c|c|c|c|c|c|c|c|c|c|}
\hline \multicolumn{11}{|c|}{ N Pondéré } \\
\hline \multirow{4}{*}{ 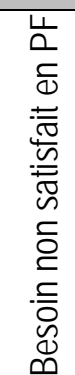 } & accès \&attitude & 60 & 287 & 177 & 118 & 128 & 131 & 89 & 231 & 64 \\
\hline & $\begin{array}{l}\text { accès, mais pas } \\
\text { d'attitude }\end{array}$ & 104 & 220 & 190 & 186 & 187 & 75 & 62 & 319 & 57 \\
\hline & $\begin{array}{l}\text { attitude, mais pas } \\
\text { d'accès }\end{array}$ & 20 & 38 & 76 & 27 & 41 & 10 & 6 & 88 & 15 \\
\hline & $\begin{array}{l}\text { ni accès ni } \\
\text { attitude }\end{array}$ & 42 & 89 & 221 & 92 & 66 & 55 & 11 & 283 & 30 \\
\hline \multirow{3}{*}{\multicolumn{2}{|c|}{$\begin{array}{l}\text { utilisation pour espacer } \\
\text { utilisation pour limiter } \\
\text { désir enfant dans }<2 \text { ans }\end{array}$}} & 72 & 422 & 82 & 96 & 167 & 174 & 153 & 108 & 69 \\
\hline & & 49 & 192 & 75 & 45 & 95 & 96 & 49 & 103 & 16 \\
\hline & & 125 & 542 & 597 & 422 & 286 & 229 & 153 & 827 & 192 \\
\hline \multicolumn{2}{|c|}{ Total } & 472 & 1790 & 1417 & 985 & 969 & 770 & 522 & 1958 & 444 \\
\hline
\end{tabular}




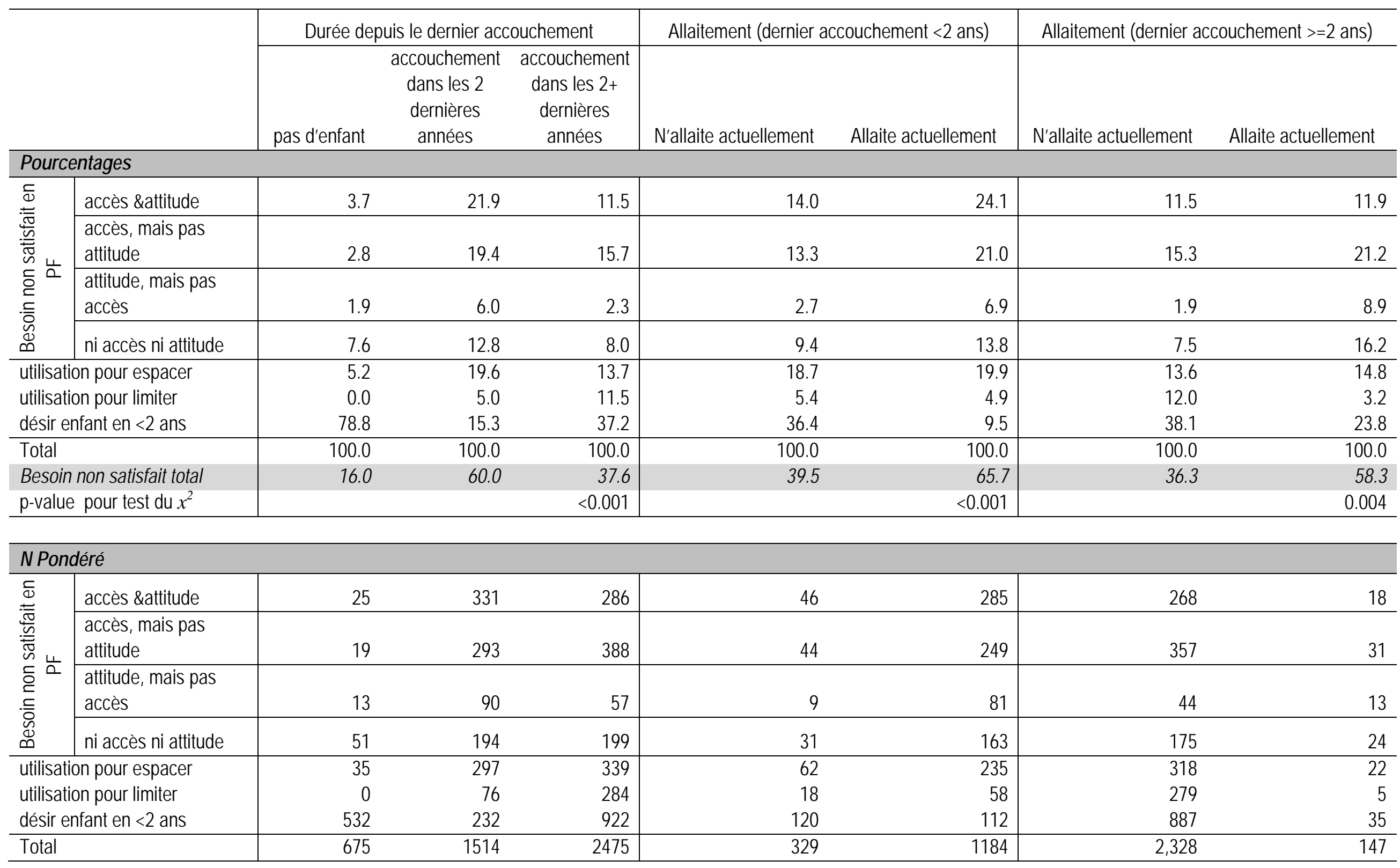




\begin{tabular}{|c|c|c|c|c|c|c|c|c|}
\hline & & \multicolumn{3}{|c|}{ Parité } & \multicolumn{2}{|c|}{ Désire un autre enfant } & \multicolumn{2}{|c|}{$\begin{array}{l}\text { A utilisé une méthode moderne dans les } 5 \\
\text { dernières années }\end{array}$} \\
\hline & & $0-2$ enfants & 3 ou 4 enfants & $5+$ enfants & Oui & Non & Non $^{\mathrm{a}}$ & Oui \\
\hline \multicolumn{9}{|c|}{ Pourcentages } \\
\hline \multirow{4}{*}{ 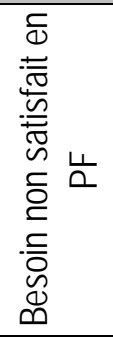 } & accès \&attitude & 12.6 & 12.7 & 16.4 & 12.9 & 17.1 & 14.1 & 13.1 \\
\hline & accès, mais pas attitude & 9.1 & 15.4 & 23.7 & 11.4 & 28.8 & 19.9 & 5.4 \\
\hline & attitude, mais pas accès & 3.0 & 3.8 & 3.8 & 3.2 & 4.3 & 5.0 & 0.3 \\
\hline & ni accès ni attitude & 7.9 & 9.4 & 12.0 & 8.7 & 12.7 & 14.0 & 0.8 \\
\hline \multirow{3}{*}{\multicolumn{2}{|c|}{$\begin{array}{l}\text { utilisation pour espacer } \\
\text { utilisation pour limiter } \\
\text { désir enfant en }<2 \text { ans }\end{array}$}} & 14.8 & 21.6 & 8.4 & 18.2 & 0.0 & 0.0 & 42.7 \\
\hline & & 0.7 & 5.5 & 20.1 & 0.0 & 37.1 & 0.0 & 22.9 \\
\hline & & 51.8 & 31.6 & 15.7 & 45.7 & 0.0 & 46.9 & 15.0 \\
\hline \multicolumn{2}{|c|}{ Total } & 100.0 & 100.0 & 100.0 & 100.0 & 100.0 & 100.0 & 100.0 \\
\hline \multicolumn{2}{|c|}{ Besoin non satisfait total } & 32.7 & 41.4 & 55.8 & 36.2 & 63.0 & 53.1 & 19.5 \\
\hline \multicolumn{2}{|c|}{$\mathrm{p}$-value pour test du $x^{2}$} & & & $<0.001$ & & $<0.001$ & & $<0.001$ \\
\hline
\end{tabular}

\begin{tabular}{|c|c|c|c|c|c|c|c|c|}
\hline \multicolumn{9}{|c|}{ N Pondéré } \\
\hline \multirow{4}{*}{ 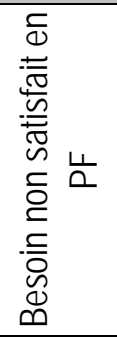 } & accès \&attitude & 272 & 137 & 233 & 475 & 167 & 437 & 205 \\
\hline & accès, mais pas attitude & 198 & 166 & 336 & 420 & 280 & 615 & 85 \\
\hline & attitude, mais pas accès & 66 & 41 & 53 & 118 & 42 & 156 & 4 \\
\hline & ni accès ni attitude & 172 & 101 & 171 & 321 & 124 & 432 & 12 \\
\hline \multirow{3}{*}{\multicolumn{2}{|c|}{$\begin{array}{l}\text { utilisation pour espacer } \\
\text { utilisation pour limiter } \\
\text { désir enfant en }<2 \text { ans }\end{array}$}} & 321 & 232 & 119 & 671 & 0 & 0 & 671 \\
\hline & & 15 & 59 & 286 & 0 & 360 & 0 & 360 \\
\hline & & 1124 & 339 & 223 & 1686 & 0 & 1450 & 235 \\
\hline \multicolumn{2}{|c|}{ Total } & 2167 & 1075 & 1421 & 3692 & 972 & 3090 & 1573 \\
\hline
\end{tabular}

a 'Non' comprend les femmes qui n'ont jamais essayé de reporter ou éviter une grossesse, les femmes qui n'ont pas utilisé de méthode modern pendant les 5 ans précédents l'enquête et celles qui avaient utilisé les méthodes traditionnelles, mais pas de méthode modern pendant 5 ans précédents l'enquête. 
Table A.4: Odds ratios ajustés pour besoin non satisfait comparé à utilisation d'une méthode moderne

\begin{tabular}{|c|c|c|c|c|c|}
\hline & \multicolumn{4}{|l|}{ SENEGAL } \\
\hline & & OR Ajusté & \multicolumn{3}{|c|}{$95 \%$ IC } \\
\hline \multicolumn{6}{|c|}{ Résidence (réf. urbain) } \\
\hline & Rural & 1.57 & 1.15 & 2.13 & ** \\
\hline \multicolumn{6}{|c|}{ Zone (réf. Ouest) } \\
\hline & Centre & 1.57 & 1.10 & 2.24 & * \\
\hline & Nord & 1.41 & 0.99 & 2.03 & \\
\hline & Sud & 0.76 & 0.50 & 1.15 & \\
\hline & Sud-est & 1.88 & 1.16 & 3.04 & ** \\
\hline \multicolumn{6}{|c|}{ Richesse (réf. Moyenne) } \\
\hline & Plus pauvre & 1.70 & 1.18 & 2.47 & ** \\
\hline & Pauvre & 1.19 & 0.86 & 1.66 & \\
\hline & Riche & 0.86 & 0.64 & 1.15 & \\
\hline & Plus riche & 0.76 & 0.52 & 1.11 & \\
\hline \multicolumn{6}{|c|}{ Instruction (réf. sans instruction) } \\
\hline & Primaire & 0.60 & 0.45 & 0.79 & $\star \star \star *$ \\
\hline & Secondaire + & 0.53 & 0.35 & 0.82 & $\star \star$ \\
\hline \multicolumn{6}{|c|}{$\begin{array}{l}\text { Allaitement (réf. pas d'allaitement au moment de } \\
\text { l'enquête) }\end{array}$} \\
\hline & Allaitement & 1.47 & 1.15 & 1.89 & ** \\
\hline \multicolumn{6}{|c|}{ Parité (réf. 3-4 enfants) } \\
\hline & 0-2 enfants & 1.51 & 1.08 & 2.10 & * \\
\hline & $5+$ enfants & 1.17 & 0.91 & 1.50 & \\
\hline \multicolumn{6}{|c|}{ Âge de la mère (réf.<30 ans) } \\
\hline & $30+$ ans & 0.83 & 0.59 & 1.16 & \\
\hline N Pondéréa & & 2977 & & & \\
\hline
\end{tabular}

a Les femmes qui désiraient un enfant dans les 2 ans et n'utilisaient pas de méthode moderne ont été exclues de cette analyse.

${ }^{*} p<0.05,{ }^{* *} p<0.01,{ }^{* \star *} p<0.001$ 
Table A.5. Rapports de risque relatif ajustés d'appartenir aux catégories spécifiées de besoin non satisfait ou de vouloir un enfant prochainement comparé aux utilisatrices de méthode moderne

\begin{tabular}{|c|c|c|c|c|c|c|c|c|c|c|c|c|c|c|c|c|c|c|c|c|}
\hline & \multicolumn{16}{|c|}{ Besoin non satisfait en PF contre utilisation d'une méthode moderne } & \multirow{2}{*}{\multicolumn{4}{|c|}{ Désir enfant dans les 2 ans }} \\
\hline & \multicolumn{4}{|c|}{ Accès \& attitude } & \multicolumn{4}{|c|}{ Accès, pas attitude } & \multicolumn{4}{|c|}{ Attitude, pas accès } & \multicolumn{4}{|c|}{$\mathrm{Ni}$ accès ni attitude } & & & & \\
\hline & $\begin{array}{l}\text { RRR }^{a} \\
\text { Ajusté }\end{array}$ & & & & $\begin{array}{l}\text { RRR } \\
\text { Ajusté }\end{array}$ & & & & $\begin{array}{l}\text { RRR } \\
\text { Ajusté }\end{array}$ & 950 & & & $\begin{array}{l}\text { RRR } \\
\text { Ajusté }\end{array}$ & 95 & & & $\begin{array}{l}\text { RRR } \\
\text { Ajusté }\end{array}$ & \multicolumn{3}{|c|}{$95 \%$ IC } \\
\hline Rural & 1.43 & 1.02 & 2.03 & * & 1.60 & 1.1 & 2.31 & * & 1.11 & 0.59 & 2.10 & & 1.81 & 1.12 & 2.93 & * & 2.68 & 1.96 & 3.67 & *** \\
\hline Centre & 1.57 & 1.03 & 2.41 & * & 1.18 & 0.79 & 1.78 & & 2.45 & 1.22 & 4.91 & * & 2.38 & 1.35 & 4.2 & ** & 1.62 & 1.13 & 2.32 & ** \\
\hline Nord & 1.17 & 0.77 & 1.79 & & 1.24 & 0.84 & 1.83 & & 1.17 & 0.50 & 2.70 & & 2.37 & 1.33 & 4.24 & ** & 1.21 & 0.84 & 1.74 & \\
\hline Sud & 1.11 & 0.70 & 1.76 & & 0.53 & 0.32 & 0.88 & * & 1.03 & 0.45 & 2.36 & & 0.71 & 0.37 & 1.35 & & 1.34 & 0.85 & 2.09 & \\
\hline Sud-est & 1.83 & 1.00 & 3.35 & & 1.35 & 0.74 & 2.47 & & 2.38 & 0.82 & 6.89 & & 3.14 & 1.53 & 6.41 & ** & 2.50 & 1.64 & 3.81 & *** \\
\hline \multicolumn{21}{|l|}{ Richesse (réf. moyenne) } \\
\hline Pplus pauvre & 1.48 & 0.94 & 2.33 & & 0.91 & 0.59 & 1.39 & & 3.98 & 2.03 & 7.83 & $\star \star \star ~$ & 2.96 & 1.76 & 4.97 & $* * *$ & 2.19 & 1.51 & 3.19 & *** \\
\hline Pauvre & 1.17 & 0.78 & 1.76 & & 1.07 & 0.72 & 1.6 & & 1.56 & 0.77 & 3.20 & & 1.35 & 0.85 & 2.16 & & 1.35 & 0.99 & 1.82 & \\
\hline Riche & 0.98 & 0.68 & 1.40 & & 0.70 & 0.46 & 1.07 & & 1.14 & 0.46 & 2.82 & & 0.96 & 0.59 & 1.56 & & 1.14 & 0.81 & 1.60 & \\
\hline Plus riche & 1.05 & 0.68 & 1.62 & & 0.59 & 0.35 & 0.99 & * & 0.57 & 0.21 & 1.55 & & 0.71 & 0.29 & 1.71 & & 1.18 & 0.76 & 1.84 & \\
\hline \multicolumn{21}{|l|}{ Instruction (réf. non instruites) } \\
\hline Primaire & 0.85 & 0.61 & 1.18 & & 0.46 & 0.33 & 0.65 & $\star \star \star ~$ & 0.39 & 0.22 & 0.70 & $\star *$ & 0.54 & 0.32 & 0.9 & * & 0.51 & 0.39 & 0.66 & *** \\
\hline Secondaire + & 0.78 & 0.49 & 1.22 & & 0.53 & 0.3 & 0.95 & * & 0.24 & 0.09 & 0.66 & $\star *$ & 0.24 & 0.12 & 0.48 & $\star \star \star ~$ & 0.34 & 0.2 & 0.57 & *** \\
\hline \multicolumn{21}{|c|}{ Allaitement (réf. pas d'allaitement au moment de l'enquête) } \\
\hline Allaitement & 1.69 & 1.25 & 2.28 & ** & 1.35 & 1.02 & 1.77 & * & 2.20 & 1.33 & 3.65 & ** & 1.12 & 0.79 & 1.59 & & 0.14 & 0.1 & 0.19 & *** \\
\hline \multicolumn{21}{|l|}{ Parité (réf 3-4 enfants) } \\
\hline $0-2$ enfants & 1.75 & 1.24 & 2.49 & ** & 1.23 & 0.83 & 1.82 & & 1.70 & 0.86 & 3.36 & & 1.73 & 0.97 & 3.09 & & 3.64 & 2.53 & 5.24 & *** \\
\hline $5+$ enfants & 1.18 & 0.83 & 1.69 & & 1.15 & 0.84 & 1.57 & & 0.84 & 0.49 & 1.44 & & 1.02 & 0.71 & 1.46 & & 0.27 & 0.19 & 0.38 & *** \\
\hline \multicolumn{21}{|l|}{ Âge de la mère (réf.<30 ans) } \\
\hline 30 +ans & 0.89 & 0.61 & 1.31 & & 1.11 & 0.76 & 1.62 & & 0.70 & 0.41 & 1.19 & & 0.69 & 0.45 & 1.07 & & 1.05 & 0.74 & 1.48 & \\
\hline $\mathrm{N}$ & & 642 & & & & 700 & & & & 160 & & & & 444 & & & & 1686 & & \\
\hline
\end{tabular}


Table A.6: Nombres de raisons de non-utilisation par femme

\begin{tabular}{rrrrrr}
\hline & \multicolumn{4}{c}{ Besoin non satisfait en planification familiale } & \\
\cline { 2 - 5 } & accès \& & & & & \\
& attitude & accès, pas attitude & attitude, pas accès & ni accès ni attitude & Total \\
\hline 0 & 14.4 & 9.9 & 11.2 & 24.0 & 14.7 \\
1 & 80.3 & 83.0 & 82.7 & 71.4 & 79.4 \\
2 & 5.1 & 6.7 & 5.7 & 3.8 & 5.4 \\
3 & 0.3 & 0.4 & 0.5 & 0.9 & 0.5 \\
\hline Total & 100.0 & 100.0 & 100.0 & 100.0 & 100.0 \\
\hline
\end{tabular}

Table A.7: Odds ratios ajustés pour ne pas avoir eu de rapports sexuels au cours des 4 dernières semaines comparé à avoir eu des rapports sexuels au cours des 4 dernières semaines

\begin{tabular}{l|l|c}
\hline & $\begin{array}{l}\text { OR } \\
\text { Ajusté }\end{array}$ & $95 \% \mathrm{IC}$ \\
\hline
\end{tabular}

Préférence en matière de fécondité et durée d'espacement préférée (réf. désire un enfant prochainement, maintenant)

\begin{tabular}{|c|c|c|c|c|c|}
\hline & ne désire aucun autre enfant & 1.53 & 1.07 & 2.18 & * \\
\hline \multicolumn{2}{|r|}{ indécise sur avoir un/un autre enfant } & 1.33 & 0.65 & 2.75 & \\
\hline \multirow{5}{*}{ 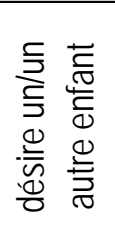 } & $<12$ mois & 1.23 & 0.30 & 5.10 & \\
\hline & 1 an & 1.09 & 0.68 & 1.73 & \\
\hline & 2 ans & 1.32 & 0.95 & 1.84 & \\
\hline & $>=3$ ans & 1.17 & 0.85 & 1.62 & \\
\hline & ne saît pas pour le moment ou une réponse non numérique & 1.75 & 1.19 & 2.58 & ** \\
\hline \multicolumn{2}{|c|}{ Situation résidentielle (vit actuellement avec mari) } & 12.30 & 9.85 & 15.36 & *** \\
\hline \multicolumn{2}{|c|}{ Utilisation d'une méthode moderne (réf. utilisatrice) } & 1.86 & 1.38 & 2.52 & *** \\
\hline Polygamie (réf. monogame) & Polygame & 1.25 & 0.98 & 1.60 & \\
\hline Instructi & $\begin{array}{r}\text { primaire } \\
\text { secondaire+ }\end{array}$ & $\begin{array}{l}1.07 \\
0.74\end{array}$ & $\begin{array}{l}0.85 \\
0.54\end{array}$ & $\begin{array}{l}1.35 \\
1.03\end{array}$ & \\
\hline Parité ( $r$ & $\begin{array}{r}3 \text { ou } 4 \text { enfants } \\
5+\text { enfants }\end{array}$ & $\begin{array}{l}0.72 \\
0.84\end{array}$ & $\begin{array}{l}0.54 \\
0.56\end{array}$ & $\begin{array}{l}0.96 \\
1.26\end{array}$ & * \\
\hline \multicolumn{2}{|c|}{ Allaitement (réf. Nallaite pas actuellement) } & 0.86 & 0.67 & 1.11 & \\
\hline \multicolumn{2}{|c|}{ Groupe d'âge (<30 ans) } & 1.19 & 0.91 & 1.55 & \\
\hline & $\mathrm{N}$ & 4659 & & & \\
\hline
\end{tabular}

a 4 femmes ont signalé qu'elles ne savaient pas le nombre d'autres femmes de leur mari

${ }^{*} p<0.05,{ }^{* *} p<0.01,{ }^{* * *} p<0.001$ 


\section{www.stepup.popcouncil.org}

Le Consortium du Programme de Recherche STEP UP (Consolider les Données Pour une Programmation sur les Grossesses Non Désirées) développe des recherches pertinentes pour les politiques afin de promouvoir une approche basée sur les évidences pour améliorer l'accès à la planification familiale et à l'avortement sans risque. STEP UP concentre ses activités dans cinq pays : le Bangladesh, le Ghana, l'Inde, le Kenya et le Sénégal.

STEP UP est coordonné par le Population Council en partenariat avec le Afrcan Population and Health Research Center ; icddr,b; le London School of Hygiene and Tropical Medicine; Marie Stopes International; et Partners in Population and Développement. STEP UP est financé par UKaid du gouvernement britannique 